\title{
Home learning environment across time: the role of early years HLE and background in predicting HLE at later ages
}

\section{published online 2019 in SCHOOL EFFECTIVENESS AND SCHOOL IMPROVEMENT https://doi.org/10.1080/09243453.2019.1618348}

ORCID Katalin Toth http://orcid.org/0000-0002-9387-3614

Pam Sammons http://orcid.org/0000-0001-6988-6784

Kathy Sylva http://orcid.org/0000-0002-1440-6277

Edward Melhuish http://orcid.org/0000-0002-2273-0894

Iram Siraj http://orcid.org/0000-0001-6752-881X

Katalin Toth a, Pam Sammons a, Kathy Sylva a, Edward Melhuish a, Iram Siraj a and Brenda Taggartb

aDepartment of Education, University of Oxford, Oxford, UK; blnstitute of Education, UCL, University of London, London, UK

\begin{abstract}
This study explores how various measures of home learning environment (HLE) collected at different ages are related to each other and explores associations when the effects of significant child and family characteristics are controlled for. Different age appropriate measures of the HLE were constructed at ages 3, 7,11, and 14. The measures were derived from parents' reports $(3,7$, and 11), but at age 14 both adolescents' self-reports and parents' questionnaires responses were used. Multilevel models tested the effects of earlier measures of HLE on later measures, while the same set of individual and family characteristics were controlled. The early years HLE measure was a significant predictor of later measures of HLE across different phases of education (primary and secondary school). The strength of the relationships between different HLE measures depended on the time lag between measurements but also on the nature of the activities covered in each dimension.
\end{abstract}

\section{KEYWORDS}

Home learning environment;

parent-child activities;

enrichment activities;

longitudinal; multilevel

modelling

\section{Introduction}

The home learning environment (HLE) has been mostly studied in the context of developmental psychology as an important factor that influences the cognitive and social development in early childhood (Bradley, 1994). The role of the home learning environment is well documented in relation to infancy developmental status (Bradley, 1994), early childhood intellectual competencies (Bradley \& Caldwell, 1982), early literacy and numeracy (Anders et al., 2012; Melhuish, 2010; Melhuish et al., 2008; Sammons, Anders, et al., 2008; Sammons et al., 2004; Van Steensel, 2006), social and behavioural development (Sylva, 
Melhuish, Sammons, Siraj-Blatchford, \& Taggart, 2010), later academic outcomes (Sammons, Sylva, Melhuish, Siraj-Blatchford, Taggart, Toth, et al., 2011;Sammons et al., 2014; Sammons, Toth, \& Sylva, 2015; Sammons, Toth, Sylva, Melhuish, et al., 2015) and to mental health (Totsika \& Sylva, 2004). Fewer studies have investigated the ways in which the home learning environment at early ages relates to later learning environment in home during childhood (Bradley, Caldwell, \& Rock, 1988; Hayes, Berthelsen, Nicholson, \& Walker, 2018; Rodriguez \& Tamis-LeMonda, 2011) and adolescence (Bradley \& Corwyn, 2001; Bradley, Corwyn, Burchinal, McAdoo, \& García Coll, 2001; Bradley et al., 2000; Vandell, Belsky, Burchinal, Steinberg, \& Vandergrift, 2010). The present study makes a new contribution to the literature by exploring the associations between various measures of the home learning environment at different time points while controlling for multiple individual and family characteristics across childhood and up to adolescence.

This study uses the social ecological theory (Bronfenbrenner, 1994) to explore the complex relationships between proximal and distal influences on home learning environment. This model views children as embedded within multiple interconnected systems, including the family, neighbourhood, school, peer culture, and community. In assessing the quality of the home learning environment, we consider the reciprocal nature of the influences between the child and his/her family (proximal influences) as well as the more distal effects like the influences of the early years home learning environment on later aspects of the learning environment as the child grows older.

The concept of home learning environment has been operationalised in various ways and levels of complexities. Kluczniok, Lehrl, Kuger, and Rossbach (2013) provided a comprehensive framework of the home learning environment by differentiating its three major components as structural characteristics (i.e., the characteristics of the family), parental educational beliefs, and the educational processes (i.e., the nature of the interactions in the family).

In this study, the theoretical construct of home learning environment (HLE) refers more specifically "to the educationally orientated activities that occur in the home environment - as opposed to learning primarily occurring in a pre-school or school setting or extension of that setting, such as school set homework, or school organised visits" (Sammons, Toth, Sylva, Melhuish, et al., 2015, p. 190). That is, the HLE is specifically conceptualised as a family- (or functional equivalent) orientated measure linking parent/carer and child interactions). For example, activities like reading to the child, support learning through play, taking the child to the library, and teaching rhymes and songs - that parents carry out with their children in order to encourage learning - were regarded as components of early years home learning environment (Melhuish et al., 2008; Sammons et al., 2004).

Family life is a dynamic process in which interactions are fluid and changes reflect the child's development, various family circumstances, and modifications in relationships (parental and siblings). Thus, it is expected that a child's experiences of the HLE would also vary over time. The characteristics of HLE in families with young children (preschool or primary school age) are well documented (Anders et al., 2012; Brooks-Gunn, Klebanov, \& Liaw, 1995; Burgess, Hecht, \& Lonigan, 2002; Melhuish et al., 2008; Sammons, Anders, et al., 2008; Sammons et al., 2004; Sénéchal \& LeFevre, 2002; Van Steensel, 2006). Less is known about the ways in which the HLE changes over time, especially in later childhood and adolescence. Studies that have explored the stability and change in aspects of the home learning environment typically considered specific child-adult activities like shared reading and its relationship to language development or literacy skills at later ages (Hayes et al., 2018; Leseman \& De Jong, 1998; Rodriguez \& Tamis-LeMonda, 2011; Rose Lehrl, Ebert, \& Weinert, 2018). Even with this particular aspect of HLE, home literacy, the findings are not always conclusive, with some studies finding that home literacy is stable over time (Leseman \& De Jong, 1998), while other studies showing that parental involvement in these activities 
declines as children grow older (Bradley et al., 2001; Hayes et al., 2018), but another suggests it can actually increase with age (Raikes et al., 2006). There is also evidence of greater variability in home literacy across time for children from disadvantaged families (Rodriguez \& Tamis-LeMonda, 2011; Rodriguez et al., 2009).

As the child develops, grows, and matures, it is likely that he/she will take an active part in shaping the nature of learning activities within the home. It is also expected that the parental level of engagement and the nature of activities would change across time (reflecting work patterns, the child's involvement in pre-school and school, plus other demands including those of siblings, and parents' perception of their child's interests and needs as they grow older). For example, Son and Morrison (2010) showed that although the HLE remained stable across time, parents were more likely to give higher importance to the HLE when children transitioned to school. Moreover, these authors found that more educated mothers or those in full-time work were more responsive to their children's needs and more likely to improve the quality of HLE when compared to those less educated or in part-time work.

The findings from past studies highlight the importance of considering individual child characteristics and family characteristics when exploring the HLE at different ages. Previous research has often focused on parents' socioeconomic status (Bradley \& Corwyn, 2002; Foster, Lambert, Abbott-Shim, McCarty, \& Franze, 2005; Kluczniok et al., 2013; Melhuish et al., 2008; Totsika \& Sylva, 2004), mother's education level (Carneiro, Meghir, \& Parey, 2013), and ethnicity (Bradley et al., 2000). Additional to these background characteristics, the present study also considers simultaneously other characteristics like mother's age, family size, and family salary.

Studies on the stability of HLE tended to focus on singular or reduced dimensions of HLE, when HLE could encompass a variety of parent-child interactions, shared activities, but also individual child activities reflecting the changing needs of children as they grow older. This paper conceptualises HLE in a broader way, considering age-appropriate activities covering not only home literacy but other enrichment and learning activities like going to the library or museums, sport, play, and computer use. It also explores the predictive value of earlier measures of HLE on later HLE measures when controlling for the effects of individual and family characteristics using multivariate statistical models. This longitudinal approach makes a new contribution to the exploration of variation in HLE experiences for different groups of children. It identifies stability and also changes in the associations with background characteristics and how they predict differences in children's and adolescents' HLE experiences at different ages over time for the same sample. It builds on earlier research that has explored change and stability in different HLE measures and associations with children's educational outcomes from pre-school through to lower secondary schooling (Bradley \& Corwyn, 2001; Sammons, Anders, et al., 2008; Sammons et al., 2004; Son \& Morrison, 2010).

Two research questions are addressed in this paper:

- How are the various measures of HLE related over time? (RQ1)

- What is the relationship between various measures of HLE while controlling for individual and family characteristics? (RQ2)

\section{Method}

Participants and procedures

The sample was drawn from the Effective Pre-school, Primary and Secondary Education Project (EPPSE 3-16) conducted in England between 1997 and 2014. EPPSE was a longitudinal study commissioned by the Department for Education (DfE). The project studied the academic and social-behavioural development of 3,172 children from preschool (age 3+ years) to age 16 . This sample also included 315 children who had not attended any pre- 
school and represented the no pre-school (home) comparison group. The characteristics of the sample and their attainments in national assessments and later in public examinations remained broadly representative of the national population of children in England across time. At age 14, the sample consisted of 2,574 students who had valid academic teacher assessment data. In total, $52 \%$ were females and $48 \%$ were males. Most of the adolescents $(74 \%)$ were of White UK heritage, while Bangladeshi young people represented the smallest ethnic group (1\%). The average age was 14.21 years $(S D=0.28)$. For more details on the sample characteristics, see Table 1 and Table 2 but also Sammons, Sylva, Melhuish, SirajBlatchford, Taggart, Toth, et al. (2011) and Sammons, Toth, Sylva, Melhuish, et al. (2015).

For the sample at age 14, we used multiple imputation to deal with missing data. The analyses conducted on the non-imputed data set led to very similar patterns of results to the ones obtained on multiple imputation data sets (see Sammons, Sylva, Melhuish, Siraj-

Blatchford, Taggart, Toth, et al., 2011). Therefore, in this paper we chose to present the set of results on the non-imputed data.

Table 1. Background characteristics of the sample at age 14 with valid academic data.

\begin{tabular}{|c|c|c|c|c|}
\hline & \multicolumn{2}{|c|}{$\begin{array}{c}\text { Age } 14 \text { English } \\
\text { Teacher Assessment } \\
\quad N=2,574 \\
\end{array}$} & \multicolumn{2}{|c|}{$\begin{array}{c}\text { Age } 14 \text { Mathematics } \\
\text { Teacher Assessment } \\
\qquad N=2,574 \\
\end{array}$} \\
\hline & $N$ & 96 & $N$ & 9 \\
\hline \multicolumn{5}{|l|}{ Gender } \\
\hline Male & 1,311 & 50.9 & 1,306 & 51.9 \\
\hline Female & 1,263 & 49.1 & 1,268 & 48.1 \\
\hline \multicolumn{5}{|l|}{ Ethnicity } \\
\hline White European & 85 & 3.3 & 85 & 3.3 \\
\hline Black Caribbean & 101 & 3.9 & 100 & 3.9 \\
\hline Black African & 53 & 2.1 & 54 & 2.1 \\
\hline Any Other Ethnic Minority & 59 & 2.3 & 59 & 2.3 \\
\hline Indian & 58 & 2.3 & 58 & 2.3 \\
\hline Pakistani & 132 & 5.1 & 125 & 4.9 \\
\hline Bangladeshi & 25 & 1.0 & 25 & 1.0 \\
\hline Mixed & 149 & 5.8 & 151 & 5.9 \\
\hline White UK & 1,911 & 74.3 & 1,916 & 74.5 \\
\hline \multicolumn{5}{|c|}{ Number of Syblings in the House (at age 3/5) } \\
\hline No siblings & 514 & 20.2 & 513 & 19.7 \\
\hline $1-2$ siblings & 1,618 & 63.7 & 1,619 & 63.6 \\
\hline $3+$ siblings & 409 & 16.1 & 409 & 16.8 \\
\hline \multicolumn{5}{|c|}{ Early Years Home Learning Environment (HLE) index } \\
\hline$<13$ & 238 & 9.6 & 235 & 10.3 \\
\hline $14-19$ & 576 & 23.1 & 576 & 25.0 \\
\hline $20-24$ & 621 & 24.9 & 623 & 23.4 \\
\hline $25-32$ & 779 & 31.3 & 783 & 29.7 \\
\hline$\geq 33$ & 278 & 11.2 & 276 & 11.6 \\
\hline
\end{tabular}

\section{Measures}

EPPSE collected a wide range of background information on the children and their family circumstances through parental interviews and questionnaires and teacher questionnaires.

Individual factors

These refer to gender and ethnicity group.

Family factors

These are in Table 2 and include mother's age, mother's education level, family salary, family socioeconomic status1 (SES), and number of siblings as reported by the parents in interviews conducted at entry to study (age 3 or 5). Less than one in five of the young people $(16 \%)$ were from large families (defined as having three or more siblings when recruited to the study), while more than half the sample had one or two siblings. 
In terms of parents' qualification levels, less than a fifth (13\%) of mothers and fathers had a degree or a higher degree. About a third (30\%) of students originated from families characterised by high socioeconomic status based on the highest of mother's or father's occupations. Half $(50 \%)$ of student's parents were classified as skilled (either skilled manual III or non-manual III), and only a very small percentage (3\%) reported they were unemployed. A fifth $(20 \%)$ of the students were eligible or receiving free school meals (FSM) at age 14. Almost half of the sample (46\%) lived in families with relatively low (below $£ 17,500)$ or no earned salary. 2

Table 2. Background characteristics of the sample at age 14.

\begin{tabular}{|c|c|c|c|c|}
\hline & \multicolumn{2}{|c|}{$\begin{array}{l}\text { Age } 14 \text { English Teacher } \\
\text { Assessment } N=2,574 \\
\end{array}$} & \multicolumn{2}{|c|}{$\begin{array}{c}\text { Age } 14 \text { Mathematics Teacher } \\
\text { Assessment } N=2,574\end{array}$} \\
\hline & $N$ & \% & $N$ & $\%$ \\
\hline \multicolumn{5}{|c|}{ Mother's Education Level (at age 3/5) } \\
\hline None & 559 & 22.3 & 558 & 223 \\
\hline Vocational & 386 & 15.4 & 385 & 15.4 \\
\hline 16 Academic & 1,002 & 40.0 & 1,004 & 40.1 \\
\hline 18 Academic & 197 & 7.9 & 197 & 7.9 \\
\hline Degree or Higher degree & 323 & 12.9 & 323 & 12.9 \\
\hline Other professional & 37 & 1.5 & 38 & 1.5 \\
\hline \multicolumn{5}{|c|}{ Father's Education Level (at age $3 / 5$ ) } \\
\hline None & 430 & 16.9 & 429 & 16.9 \\
\hline Vocational & 308 & 12.1 & 307 & 12.1 \\
\hline 16 academic & 623 & 24.5 & 624 & 24.6 \\
\hline 18 academic & 179 & 7.1 & 178 & 7.0 \\
\hline Degree or Higher degree & 333 & 13.1 & 337 & 133 \\
\hline Other professional & 29 & 1.1 & 29 & 1.1 \\
\hline Absent Father & 637 & 25.1 & 635 & 25.0 \\
\hline \multicolumn{5}{|l|}{ Family Highest SES (at age 3/5) } \\
\hline Professional Non-Manual & 165 & 6.5 & 167 & 6.6 \\
\hline Other Professional Non-Manual & 605 & 23.9 & 605 & 23.9 \\
\hline Skilled Non-Manual & 876 & 34.6 & 879 & 34.7 \\
\hline Skilled Manual & 398 & 15.7 & 395 & 15.6 \\
\hline Semi-Skilled & 349 & 13.8 & 348 & 13.7 \\
\hline Unskilled & 68 & 2.7 & 68 & 2.7 \\
\hline Unemployed/Not working & 73 & 2.9 & 72 & 2.8 \\
\hline \multicolumn{5}{|l|}{ Free School Meal (at age 14) } \\
\hline No FSM & 2,041 & 80.2 & 2,040 & 80.2 \\
\hline FSM & 504 & 19.8 & 504 & 19.8 \\
\hline \multicolumn{5}{|l|}{ Family Eamed salary (at age 7) } \\
\hline No salary & 488 & 24.2 & 485 & 24.1 \\
\hline$£ 2,500-17,499$ & 442 & 21.9 & 440 & 21.8 \\
\hline$£ 17,500-29,999$ & 375 & 18.6 & 375 & 18.6 \\
\hline$£ 30,000-37,499$ & 245 & 12.2 & 246 & 12.2 \\
\hline$£ 37,500-67,499$ & 375 & 18.6 & 379 & 18.8 \\
\hline$\{67,500-132,000+$ & 90 & 4.5 & 90 & 4.5 \\
\hline
\end{tabular}

Parent-child activities and routines during the preschool years were aggregated into a scale, while those measured during lower primary school at age 7, upper primary school at age 11, and lower secondary school at age 14 were combined into multiple factors. Means and standard deviations for the continuous measures are in Table 3.

Early years HLE.

The indicator of the HLE developed by EPPSE in early years was based on the frequency of specific activities involving the child as reported by parents during the pre-school period (i.e., teaching the child the alphabet, playing with letters and numbers, library visits, reading to the child, teaching the child songs or nursery rhymes, etc.). These measures were combined to form an overall early years HLE index (Melhuish et al., 2008; Sammons, Anders, et al., 2008; Sammons et al., 2004). The early years home learning scale has a range of 0 to 45 ; the higher the value, the better the quality of the early years HLE. The Cronbach's $\alpha$ for this 
scale is 0.59 . We then split the scale into five HLE categories by selecting the top and bottom $10 \%$ and then splitting the remaining $80 \%$ in roughly three groups for medium low, medium, and medium high HLE (Sammons, Toth, Sylva, Melhuish, et al., 2015). In earlier research on the EPPSE sample, it was hypothesized that threshold effects might have operated; therefore, the comparisons were made on the effects of high and low HLE on child outcomes. These were then directly compared to the effects of high and low parental qualifications as background characteristics were categorical. In this paper, the same HLE categories were used for comparison. Over four out of $10(43 \%)$ of the adolescents had experienced a good or very good home learning environment in the early years. Less than one in $10(9 \%)$ of the current sample had a very poor early years HLE.

HLE during lower primary school (age 7).

Parents were surveyed by questionnaires during lower primary school (age 6-7 years) about their interactions with their child at home. They reported on the frequency of reading to/with the child, taking the child out on educational visits, computing activities, sport activities, dance, and so forth. Confirmatory factor analyses indicated that the individual HLE measures linked to four latent factors3: home computing (Cronbach's $\alpha=0.77$ ), one-to-one interaction (Cronbach's $\alpha=0.58$ ), expressive play (Cronbach's $\alpha=0.54$ ), and enrichment outings (Cronbach's $\alpha=0.51$ ) (Sammons, Sylva, Melhuish, Siraj-Blatchford, Taggart, \& Hunt, 2008; Sammons, Sylva, Melhuish, Siraj-Blatchford, Taggart, \& Jelicic, 2008).

HLE during upper primary school (age 11).

When children were age 11, parents were again surveyed to report on activities such as the frequency of internet usage, taking the child out to physical activities and educational visits, computing activities, and teaching the child different subjects. Four HLE factors were extracted using exploratory and confirmatory factor analyses3: educational computing (Cronbach's $\alpha=0.83$ ), parent-child learning processes (Cronbach's $\alpha=0.56$ ), individual child activities (Cronbach's $\alpha=0.51$ ),

and computer games (Cronbach's $\alpha=0.56$ ) (Sammons, Sylva, Melhuish, Siraj-Blatchford, Taggart, Draghici, et al., 2011).

HLE during lower secondary school (age 14).

The HLE measure at age 14 incorporates information sourced not just from the parent but from the adolescents themselves. This innovation was designed to take account of the greater independent influence the adolescent, at age 14, could exert over their own home learning environment. Five HLE

factors were extracted from the individual items using exploratory and confirmatory factor analyses3: learning support and resources (parents' reports, Cronbach's $\alpha=0.68$ ), computer use (students' report, Cronbach's $\alpha=0.68$ ), parental interest in school (parents' reports, Cronbach's $\alpha=0.64$ ), academic enrichment (students' report, Cronbach's $\alpha=0.46$ ), and parental academic supervision (students' report, Cronbach's $\alpha=0.56$ ) (Sammons et al., 2014).

Each of these latent factors measured at age 7,11 , and 14 were then categorised into low (group of children with scores below one standard deviation from the mean), medium (group of children with scores between one standard deviation below and above the mean), and high (group of children with scores above one standard deviation from the mean). 4

\section{Analysis strategy}

Correlations were used to explore the relationships between different measures of HLE at various time points. Then, multilevel models were used to take account of clustering in the EPPSE data set and reflecting the distribution of the sample at different ages in institutional grouping like primary schools and secondary schools. The categorical version of the early years HLE measure was used as a predictor for later HLE measures at age 7,11 , and 14, 
while controlling for the effects of various child, family, and neighbourhood background characteristics. Similarly, the categorical versions of the HLE dimensions at age 7 and 11 were used as predictors for the later HLE dimensions at age 11 and 14, while controlling for the effects of the same set of background characteristics.

Table 3. Means and standard deviations of the continuous HLE measures at different ages.

\begin{tabular}{llll}
\hline & $N$ & Mean & \\
\hline Early years HLE & 3,069 & 22.8 & 7.8 \\
Home computing at age 7 & 2,251 & 1.9 & 0.9 \\
Interactions at age 7 & 2,312 & 1.6 & 0.3 \\
Enrichment out ing at age 7 & 2,300 & 1.1 & 0.3 \\
Expressive play at age 7 & 2,373 & 1.8 & 0.4 \\
Educational computing at age 11 & 1,927 & 2.4 & 0.9 \\
Interactive leaming processes at age 11 & 1,927 & 1.9 & 0.5 \\
Individual child activities at age 11 & 1,927 & 1.4 & 0.3 \\
Computer games at age 11 & 1,927 & 1.6 & 0.8 \\
Academic enrichment at age 14 & 1,711 & 2 & 1.6 \\
Parental interest at age 14 & 1,655 & 7.2 & 1.3 \\
Computer use at age 14 & 1,706 & 7.9 & 3.1 \\
Learning support at age 14 & 1,628 & 4.5 & 2.1 \\
Parental academic supervision at age 14 & 1,745 & 5.1 & 1.1 \\
\hline
\end{tabular}

\begin{tabular}{|c|c|c|c|c|c|c|c|c|c|c|c|c|c|}
\hline & \multicolumn{4}{|c|}{ HLE factors at age 7} & \multicolumn{4}{|c|}{ HLE fadors at age 11} & \multicolumn{4}{|c|}{ HLE factors at age 14} & \multirow[b]{2}{*}{ Supervi: } \\
\hline & $\begin{array}{l}\text { Home } \\
\text { computing }\end{array}$ & Irteractions & $\begin{array}{l}\text { Emichment } \\
\text { outings }\end{array}$ & $\begin{array}{c}\text { Expressive } \\
\text { play }\end{array}$ & $\begin{array}{l}\text { Educational } \\
\text { cmputing }\end{array}$ & $\begin{array}{l}\text { Irteractive } \\
\text { learning } \\
\text { proosses }\end{array}$ & $\begin{array}{l}\text { Individual } \\
\text { child } \\
\text { adivities }\end{array}$ & $\begin{array}{l}\text { Computer } \\
\text { games }\end{array}$ & Enrichment & Interest & Computer & Support & \\
\hline Early years HLE & $0.13^{* * *}$ & $0.29+2 *$ & $036^{* * *}$ & $0.22 * *$ & $0.19 * *$ & $0.32^{* *}$ & $0.31=$ & $-0.07^{*}$ & $0.16^{* * *}$ & $0.19 * * *$ & $0.10^{* 2 *}$ & $0.10^{* 2 *}$ & 0.02 \\
\hline Home computing age? & & & & & $0.22^{20}$ & $0.14^{* \cdots}$ & oos & $0.15 * * t$ & 0.01 & $0.09^{*}$ & 0.03 & $0.16^{* * *}$ & $0.08^{\circ}$ \\
\hline Interactions age 7 & & & & & $0.10^{21 .}$ & $0.26 * 0$ & $022 \ldots$ & -0.04 & $0.14 * \ldots$ & $0.20^{* * *}$ & -0.02 & Q.19.:. & $0.09 *$ \\
\hline Enrichment age 7 & & & & & $0.21=$ & $0.40 * \cdots$ & $0.25=$ & 0.04 & $0.17 * *$ & $0.17 * *$ & 0.04 & $0.21 \cdots$ & $0.0 \mathrm{~s}^{*}$ \\
\hline Expressive play age 7 & & & & & $0.13^{m-*}$ & $0.20^{\circ-0}$ & $0.43 m$ & $-0.11^{* * *}$ & $0.09 *$ & $0.08^{* *}$ & 0.07 & $0.06^{\circ}$ & 0.05 \\
\hline $\begin{array}{l}\text { Eduational } \\
\text { computing age } 11\end{array}$ & & & & & & & & & 0.00 & $0.1 \mathrm{en}^{2 * *}$ & $0.15 * *$ & $0.26 * *$ & $0.07 \%$ \\
\hline $\begin{array}{l}\text { Irteractive learning } \\
\text { processes age } 11\end{array}$ & & & & & & & & & $0.16 * *$ & $0.23 \% *$ & 0.03 & $0.290 *$ & $0.12 *$ \\
\hline $\begin{array}{l}\text { Individual child } \\
\text { activities age } 11\end{array}$ & & & & & & & & & $0.23 * *$ & $0.17 \times *$ & $0.0 \%$ & $0.21 * *$ & 0.05 \\
\hline $\begin{array}{l}\text { Computer games } \\
\text { age } 11\end{array}$ & & & & & & & & & $-0.13 * 1$ & 0.04 & 0.00 & $0.0 \%$ & 0.02 \\
\hline
\end{tabular}

\section{Results Home learning environment across time - correlational findings (RQ1)}

The results of correlational analyses showed that the various dimensions of HLE were moderately associated with each other over time (see Table 4). The early years HLE measure was positively and statistically significantly associated with the HLE factors at age 7,11 , and14. The overall index of early HLE was significantly associated with parent-child enrichment outing/activity outside home $(r=0.36)$ at age 7 , parent-child interactive learning processes $(r=0.32)$ at age 11 , but more weakly to the academic enrichment measure $(r=$ 
0.16) at age 14, suggesting that parents who engage in learning activities when the children are young continue to be involved later as they go through primary and secondary school (although the frequency of these activities do vary across time). This tendency is also highlighted by the statistically significant associations between parent-child enrichment outing/activity outside home at age 7 and parent-child interactive learning processes at age $11(r=0.40)$. Activities children do on their own over time were also statistically significantly associated; expressive play at age 7 was moderately correlated with individual child activities at age $11(\mathrm{r}=0.43)$. Parents who reported they interacted more often with their child on a one-to-one basis during lower primary school (at age 7) doing activities like reading or listening to the child reading tended to help teach a school subject and join the child during games or play during upper primary school $(r=0.26)$ and were more likely to report talking about school work and their children's experiences at school when they moved into secondary education at age $14(r=0.20)$. Measures of computer usage were also associated across time. Parents and children who engaged in home computing activities during the first years of primary school were more likely to report the use of computers for educational purposes $(r=0.22)$ and computer games $(r=0.15)$ at age 11 . The same measure was not significantly associated with the computer use in secondary school as the later factor is a relatively specific measure of using the computer for emails or browsing the internet, while the earlier measures include broader computer-based activities. While expressive play at age 7 was significantly and negatively associated with computer games $(r=-0.11)$ at age 11 , it was positively associated with computer use at age $14(r=0.07)$. Parent - child educational computing was significantly associated with computer use at age $14(r=0.15)$.

Early years HLE as a predictor of later measures of HLE, controlling for background (RQ2) The correlational associations described above did not take into account the relative influence of other background characteristics. Multilevel modelling provides more detailed results of the net contribution of HLE measures, whilst controlling for individual and family characteristics, and so enables the identification of the strongest net predictors over time. Results are reported in effect sizes5 (ES), a statistical measure of the relative strength of different predictors to facilitate comparisons across various models at different child ages.

The early years HLE was found to be a significant predictor of the later primary and secondary HLE measures after controlling for associations with other significant child and family characteristics (see Tables 5, 6 and 7). The early years HLE index significantly predicted all four measures of HLE in lower primary school (age 7). Higher early years HLE scores was the strongest predictor of the more frequent parent-child one-to-one interactions at home at ages $7(E S=0.69)$ and $11(E S=0.71)$ beyond other individual and family influences such as SES, salary, parents' education level, and so forth. Parents who frequently interacted with their children at preschool age were more likely to take them out for museum and library visits ( $E S=0.91)$ or use the computer with them for educational purposes at age $7(E S=0.44)$. Higher early HLE scores also predicted greater parental interest in their children's later secondary school experiences at age 14 (ES=0.31). Children who experienced a very high early years HLE were significantly more likely to engage in expressive play at age 7 ( $E S=0.65)$, in individual activities at age $11(E S=0.56)$, and in enrichment activities at age $14(E S=0.35)$. The findings reveal that the relationship between the early years HLE and later manifestations of home learning linked to parent-child interactions tends to be sustained over time. Thus, when the child was older, at age 11, the early years HLE remained the strongest predictor of interactive learning processes in upper 
primary school ( $E S=0.71)$. However, it is important to note that the early years HLE did not predict the child's involvement in computer games at age 11. This suggests that computer may be viewed differently in relation to learning at home. At the time of the research when children were in primary school (at the turn of the millennium in the early 2000s), there were rapid developments in IT availability and use at home. This may have shaped family and children's activities and changes in the ways they use computers over time. On the other hand, the early years HLE was a positive and significant predictor of parents' reported engagement with the child in educational computing at age $11(E S=0.42)$ and in lower secondary school of computer use (internet) at age 14 (ES=0.47).

HLE measures at age 7 as predictors of later measures of HLE Parents who took their children to educational visits to museums or parks at age 7 , were also more likely to engage in interactive learning activities at age 11(ES=1.04) and use the computer as a learning resource at age 11 and 14, while the children were significantly more likely to read and use the library at age 11 and 14 (see Tables 6 and 7). Similarly, children who engaged in expressive play at age 7 continued to participate in individual activities at age 11 ( $E S=0.88$ ). As expected, high levels of parent-child one-to-one interactions at age 7 significantly predicted subsequent parent-child interactive learning processes at age $11(E S=0.64)$ and children's individual activities ( $E S=0.39$ ). It also predicted, but to a lesser extent, parental academic supervision ( $E S=0.48$ ), parental interest in school $(E S=0.42)$, and parental support $(E S=0.39)$ in lower secondary education when the sample were age 14. Parents' using the computer at home with their 7-year-old child was a statistically significant predictor of using the computer for educational purposes $(E S=0.60)$ or for games $(E S=0.38)$ when the child was older, at 11 , or of parents providing more learning support and resources at age 14 $(\mathrm{ES}=0.35)$. 
Table 5. Contextualised models for lower primary school measures of HLE at age 7.

\begin{tabular}{|c|c|c|c|c|c|c|c|c|c|c|c|c|c|c|c|c|}
\hline & \multicolumn{4}{|c|}{ Home computing at age 7} & \multicolumn{4}{|c|}{ Enrichment outings at age 7} & \multicolumn{4}{|c|}{ Interactions at age 7} & \multicolumn{4}{|c|}{ Expressive play at age 7} \\
\hline & Coeff. & $\begin{array}{l}\text { Std. } \\
\text { Erro }\end{array}$ & $\begin{array}{l}\text { Effect } \\
\text { Size }\end{array}$ & Sig. & Coefl. & $\begin{array}{l}\text { Std } \\
\text { Enror }\end{array}$ & $\begin{array}{l}\text { Effect } \\
\text { Seze }\end{array}$ & Sig & Coell. & $\begin{array}{l}\text { Std. } \\
\text { End }\end{array}$ & $\begin{array}{l}\text { Effect } \\
\text { Sue }\end{array}$ & Sig & Coeff. & $\begin{array}{l}\text { Std. } \\
\text { Error }\end{array}$ & $\begin{array}{l}\text { Effect } \\
\text { Size }\end{array}$ & Sig \\
\hline \multicolumn{17}{|l|}{ Eanty Years MLE (Red = 0-13) } \\
\hline $14-19$ & 0.10 & 0.08 & 0.11 & & 0.12 & 0.02 & 0.42 & $\leftrightarrow$ & 0.05 & 0.03 & 0.18 & * & 0.10 & 0.03 & 0.29 & $\omega$ \\
\hline $20-24$ & 0.19 & 0.08 & 0.21 & - & 0.18 & 0.02 & 0.65 & $\omega$ & Q.14 & 0.03 & 0.46 & $\omega$ & 0.17 & 0.03 & 0.50 & $\omega$ \\
\hline $25-32$ & 0.27 & 0.08 & 0.29 & $\omega$ & 0.22 & 0.02 & 0.76 & $\omega$ & 0.17 & 0.03 & 0.56 & $\cdots$ & 0.16 & 0.03 & 0.48 & $\omega$ \\
\hline$>33$ & 0.41 & 0.10 & 0.44 & $\omega$ & 0.26 & 0.03 & 0.91 & $\omega$ & 0.21 & 0.03 & 0.69 & $\omega$ & 0.22 & 0.03 & 0.65 & $\omega$ \\
\hline $\begin{array}{l}\text { Gris or Gender Ref=Boys) } \\
\text { Ethic group (Ref = Whis ub }\end{array}$ & $\begin{array}{l}-0.31 \\
\text { trage) }\end{array}$ & 0.04 & -0.34 & $\cdots$ & -0.05 & 0.01 & \multicolumn{10}{|c|}{ Ethinic group (Ref = Whise UK Hestroge) } \\
\hline White European Heritage & -0.07 & 0.11 & -0.07 & & 0.04 & 0.03 & 0.14 & & 0.01 & 0.04 & 0.05 & & 0.08 & 0.04 & 0.23 & $\cdot$ \\
\hline Black Carlbbean Heritage & 0.25 & 0.12 & 0.27 & * & -0.13 & 0.04 & -0.45 & $\cdots$ & -0.02 & 0.04 & -0.06 & & 0.02 & 0.04 & 0.05 & \\
\hline Wad African Heritage & 0.65 & 0.18 & 0.71 & $* *$ & -0.05 & 0.05 & -0.18 & & -0.01 & 0.06 & -0.03 & & 0.10 & 0.06 & 0.31 & \\
\hline Any Other Ethric Minority & -0.08 & 0.14 & -0.09 & & -0.08 & 0.04 & -0.28 & & -0.09 & 0.05 & -0.31 & - & 0.03 & 0.05 & 0.10 & \\
\hline Indian & 0.23 & 0.16 & 0.25 & & -0.05 & 0.05 & -0.16 & & -0.06 & 0.05 & -0.20 & & 0.08 & 0.05 & 0.25 & \\
\hline Pakistani & -0.31 & 0.12 & -0.33 & * & -0.21 & 0.03 & -0.73 & $m$ & -0.17 & 0.04 & -0.56 & $\cdots$ & -0.14 & 0.04 & -0.40 & *.. \\
\hline Bangladeshi & -0.09 & 0.24 & -0.10 & & -0.23 & 0.08 & -0.81 & $*$ & -0.08 & 0.08 & -0.26 & & -0.12 & 0.09 & -0.34 & \\
\hline Mbed Race & 0.10 & 0.09 & 0.11 & & 0.05 & 0.03 & 0.19 & & 0.02 & 0.03 & 0.06 & & 0.02 & 0.03 & 0.097 & \\
\hline \multicolumn{17}{|c|}{ Number of siblings at age $3 / 5$ Ref $=N b$ Solings) } \\
\hline 1 sibing & 0.01 & 0.06 & 0.01 & & -0.00 & 0.02 & -0.01 & & -0.03 & 0.02 & -0.11 & & 0.00 & 0.02 & 0.01 & \\
\hline 2 sibings & 0.04 & 0.06 & 0.04 & & -0.04 & 0.02 & -0.15 & • & -0.03 & 0.02 & -0.10 & & -0.02 & 0.02 & -0.06 & \\
\hline \multicolumn{17}{|c|}{ Parents' highest SES at age 3/5 (Ref = Professional, Non-Manual) } \\
\hline $\begin{array}{l}\text { Other protessional non- } \\
\text { manual }\end{array}$ & 0.22 & 0.08 & 0.26 & $*$ & -0.01 & 0.02 & -0.02 & & -0.01 & 0.03 & -0.04 & & -0.03 & 0.03 & -0.08 & \\
\hline Skilled non-manual & 0.17 & 0.09 & 0.18 & & -0.02 & 0.03 & -0.07 & & -0.05 & 0.03 & -0.16 & & -0.05 & 0.03 & -0.14 & \\
\hline Skilled manual & 0.17 & 0.10 & 0.18 & & -0.04 & 0.03 & -0.15 & & -0.04 & 0.03 & -0.12 & & -0.04 & 0.03 & -0.11 & \\
\hline Semiskilled & 0.03 & 0.10 & 0.03 & & -0.12 & 0.03 & -0.41 & $\cdots$ & -0.08 & 0.03 & -0.27 & • & -0.03 & 0.04 & -0.08 & \\
\hline Unasilled & -0.45 & 0.18 & -0.48 & • & -0.18 & 0.05 & -0.63 & $\cdots$ & -0.24 & 0.06 & -0.30 & $\cdots$ & 0.01 & 0.06 & 0.03 & \\
\hline Never worked & 0.23 & 0.18 & 0.25 & & -0.02 & 0.05 & -0.07 & & 0.03 & 0.06 & 0.11 & & -0.14 & 0.06 & -0.41 & $\cdot$ \\
\hline \multicolumn{17}{|c|}{ Fanily salay at age 7 (fief $=$ No Salary) } \\
\hline$£ 2,500-15,000$ salary & -0.11 & 0.06 & -0.12 & & 0.03 & 0.02 & 0.09 & & -0.03 & 0.02 & -0.11 & & -0.02 & 0.02 & -0.06 & \\
\hline $617,500-27,500$ salary & 0.01 & 0.07 & 0.01 & & 0.03 & 0.02 & 0.12 & & -0.05 & 0.02 & -0.17 & - & -0.02 & 0.02 & -0.06 & \\
\hline $630,000-35,000$ salay & 0.10 & 0.08 & 0.11 & & 0.03 & 0.02 & 0.12 & & -0.06 & 0.02 & -0.19 & - & -0.01 & 0.03 & -0.04 & \\
\hline$\{37,500-66,000$ salary & 0.12 & 0.07 & 0.13 & & 0.06 & 0.02 & 0.20 & * & -0.02 & 0.02 & -0.07 & & -0.02 & 0.02 & -0.07 & \\
\hline$\{67,500-132,000$ salary & 0.13 & 0.10 & 0.14 & & 0.02 & 0.03 & 0.06 & & -0.05 & 0.03 & -0.16 & & -0.08 & 0.04 & -0.23 & * \\
\hline \multicolumn{17}{|c|}{ Mother's highest qualificatons level at age 3/5 Ref = None) } \\
\hline
\end{tabular}

(Continued)

Table 5. Continued

\begin{tabular}{|c|c|c|c|c|c|c|c|c|c|c|c|c|c|c|c|c|}
\hline & \multicolumn{4}{|c|}{ Hame computing at age 7} & \multicolumn{4}{|c|}{ Enrichment outings at age 7} & \multicolumn{4}{|c|}{ Interactions at age 7} & \multicolumn{4}{|c|}{ Expressive play at age 7} \\
\hline & Coeff. & $\begin{array}{l}\text { Std. } \\
\text { Eror }\end{array}$ & $\begin{array}{l}\text { Effect } \\
\text { Size }\end{array}$ & Sig. & Coeff. & $\begin{array}{l}\text { Std } \\
\text { Erro }\end{array}$ & $\begin{array}{l}\text { Effect } \\
\text { Size }\end{array}$ & Sig & Coeft. & $\begin{array}{l}\text { Std. } \\
\text { Enor }\end{array}$ & $\begin{array}{l}\text { Effect } \\
\text { Scze }\end{array}$ & Sig & Coeff. & $\begin{array}{l}\text { Std. } \\
\text { Enor }\end{array}$ & $\begin{array}{l}\text { Effect } \\
\text { Sane }\end{array}$ & Sig \\
\hline Vocational & 0.13 & 0.08 & 0.15 & & 0.10 & 0.02 & 0.35 & $* *$ & 0.07 & 0.02 & 0.25 & $*$ & 0.02 & 0.03 & 0.05 & \\
\hline Academic age 16 & 0.04 & 0.06 & 0.04 & & 0.11 & 0.02 & 0.39 & $* *$ & 0.07 & 0.02 & 0.24 & $\cdots$ & 0.04 & 0.02 & 0.13 & - \\
\hline Academicage 18 & 0.04 & 0.09 & 0.04 & & 0.12 & 0.03 & 0.41 & w* & 0.09 & 0.03 & 0.31 & $*$ & 0.05 & 0.03 & 0.14 & \\
\hline Degree or higher degree & 0.04 & 0.09 & 0.04 & & 0.17 & 0.03 & 0.59 & 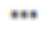 & 0,0 & 0.03 & 0.31 & $*$ & 0.05 & 0.03 & 0.16 & \\
\hline Other professional & -0.02 & 0.17 & -0.03 & & 0.12 & 0.05 & 0.43 & • & 0.06 & 0.05 & 0.21 & & 0.03 & 0.06 & 0.10 & \\
\hline Mother's age & -0.12 & 0.04 & -0.17 & $*$ & 0.03 & 0.01 & 0.14 & $*$ & 0.01 & 0.01 & 0.03 & & $-0,0$ & 0.01 & -0.09 & \\
\hline Intercept & 1.66 & 0.13 & & w. & 0.85 & 0.04 & & $\omega$ & 150 & 0.04 & & *.* & 156 & 0.05 & & ... \\
\hline Varlance Level 2 & 0.03 & 0.01 & & w. & 0.00 & 0.00 & & $* *$ & 0.01 & $0 . \infty$ & & $* *$ & $\infty \infty$ & 0,0 & & $\cdots$ \\
\hline Varlance Level 1 & 0.85 & 0.03 & & w. & 0.08 & 0.00 & & w. & 0.09 & 0.00 & & $\cdots$ & 0.11 & 0,0 & & $\ldots$ \\
\hline Total Variance & 0.88 & & & & 0.08 & & & & 0.10 & & & & 0.12 & & & \\
\hline Number of Observations & 2209 & & & & 2256 & & & & 2269 & & & & 2,327 & & & \\
\hline Number of Groups & 741 & & & & 78 & & & & 749 & & & & 755 & & & \\
\hline $\begin{array}{l}\text { Deviance ( }-2 \times \text { Log } \\
\text { Restricted Likelihood) }\end{array}$ & 5987.90 & & & & 722.86 & & & & $1,107,37$ & & & & 1,58051 & & & \\
\hline Intra Class Correlation 0 (c) & 0.036 & 0.016 & & • & 0.016 & 0.014 & & & 0.073 & 0.017 & & $\cdots$ & 0.015 & 0.013 & & \\
\hline $\begin{array}{l}\text { \%o feduction Student } \\
\text { Variance }\end{array}$ & 7.02 & & & & 14.73 & & & & 8.66 & & & & 16.17 & & & \\
\hline \% Reduction School Varlance & 37.82 & & & & 91.31 & & & & 46.29 & & & & 35.40 & & & \\
\hline \% fleducion Total Variance & 8.66 & & & & 25.02 & & & & 13.11 & & & & 16.55 & & & \\
\hline
\end{tabular}


Table 6. Contextualised models for upper primary school measures of HLE at age 11.

\begin{tabular}{|c|c|c|c|c|c|c|c|c|c|c|c|c|c|c|c|c|}
\hline & \multicolumn{4}{|c|}{ Educational computing at age 11} & \multicolumn{4}{|c|}{$\begin{array}{l}\text { Irteractive learning processes at age } \\
\qquad 11\end{array}$} & \multicolumn{4}{|c|}{ Individual activities at age 11} & \multicolumn{4}{|c|}{ Computer games at age 11} \\
\hline & Coeff. & $\begin{array}{l}\text { Std. } \\
\text { Error }\end{array}$ & $\begin{array}{l}\text { Effect } \\
\text { Size }\end{array}$ & Sig. & Coeff. & $\begin{array}{l}\text { Std. } \\
\text { Error }\end{array}$ & $\begin{array}{l}\text { Effect } \\
\text { Size }\end{array}$ & Sig. & Coeff. & $\begin{array}{l}\text { Stid. } \\
\text { Errox }\end{array}$ & $\begin{array}{l}\text { Effed } \\
\text { Size }\end{array}$ & Sig. & Coeff. & $\begin{array}{l}\text { Std. } \\
\text { Error }\end{array}$ & $\begin{array}{l}\text { Effect } \\
\text { Sire }\end{array}$ & Sig \\
\hline \multicolumn{17}{|l|}{ Early Years HIE (Ref $=0-13)$} \\
\hline $14-19$ & 0.17 & 0.11 & 0.20 & & 0.10 & 005 & 0.28 & - & 0.03 & 0.58 & 0.13 & & 0.03 & 0.09 & 004 & \\
\hline $20-24$ & 0.29 & 0.11 & 0.34 & $\boldsymbol{*}$ & 0.19 & 0.05 & 0.51 & $\cdots$ & 0.09 & 0.04 & 0.34 & * & 0.04 & 0.09 & 0.05 & \\
\hline $25-32$ & 0.31 & 0.11 & 0.37 & *. & 0.23 & 005 & 0.62 & $\cdots$ & 0.13 & 0.04 & 0.49 & $m$ & -0.02 & 0,09 & $-0,02$ & \\
\hline $\begin{array}{l}>33 \\
\text { HLE Home Compuring age } 7 \text { (Ref: }\end{array}$ & $=\begin{array}{r}0.35 \\
\text { Low }\end{array}$ & 0.12 & 0.42 & $*$ & 0.27 & 0.05 & \multicolumn{9}{|c|}{ HLE Home Computing age 7 (Ref $=$ Low) } & \\
\hline $\begin{array}{l}\text { HLF Home Computing } \\
\text { Medium }\end{array}$ & 0.29 & 006 & 0.35 & $\cdots$ & 0.05 & 003 & 0.13 & & 0.02 & $0 \leqslant 2$ & 0.86 & & 0.14 & 0.06 & 020 & $*$ \\
\hline $\begin{array}{l}\text { HE Home Computing High } \\
\text { HE Interadion age } 7 \text { (Ref }=\text { Low) }\end{array}$ & 0.50 & 008 & 0,60 & $\cdots$ & 0.11 & 004 & 030 & ". & 0.04 & 0.018 & 0.14 & & 0.28 & 0,07 & 038 & $\cdots$ \\
\hline HLE Interaction Medium & 0.96 & 007 & 0,07 & & 0.09 & 003 & 0.24 & 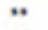 & 0.04 & 0.12 & 0.16 & & -0.12 & 0.06 & -0.17 & - \\
\hline $\begin{array}{l}\text { HLE irteraction High } \\
\text { HLE Enridiment Outings oge } 7 \text { (Re }\end{array}$ & $\begin{array}{c}0.17 \\
\text { ef }=\text { Low }\end{array}$ & 0.11 & 0.20 & & 0.24 & 0.05 & 0.64 & $\cdots$ & 0.11 & 0.04 & 0.39 & " & 0.03 & 0.09 & 005 & \\
\hline $\begin{array}{l}\text { HLE Enrichment Outings } \\
\text { Medium }\end{array}$ & 0.24 & 008 & 0.28 & $*$ & 0.25 & 0.04 & 0.67 & $\cdots$ & 0.08 & $0 / 8$ & 0.30 & $*$ & 0.10 & 0.07 & 0.14 & \\
\hline $\begin{array}{l}\text { HLE Enrichment Outings } \\
\text { High }\end{array}$ & 0.37 & 0.11 & 0,44 & $\cdots$ & 0.39 & 0.05 & 1.04 & $\cdots$ & 0.13 & $0.5 \mathrm{~A}$ & $0.4 B$ & $\cdots$ & 0.22 & 0.10 & 031 & - \\
\hline \multicolumn{17}{|c|}{ HEF Expressive Play age 7 (hef $=$ Low) } \\
\hline HLE Play Medium & 0.09 & 0.07 & 0.11 & & 0.10 & 0.03 & 0.27 & $\boldsymbol{*}$ & 0.18 & 0.02 & 0.64 & ... & -0.11 & 0.06 & -0.15 & \\
\hline HLE Pay High & 0.12 & 009 & 0.14 & & 0.11 & 0.04 & 0.29 & $*$ & 0.24 & 0.03 & 0.88 & $\cdots$ & -0.08 & $0.0 \mathrm{a}$ & -0.11 & \\
\hline Girts $\alpha$ Gender (Ref=Eoys) & 0.05 & 005 & 0.06 & & 004 & $a m$ & 0.12 & - & 0.18 & 0,12 & 0.57 & $\cdots$ & -0.43 & 0,04 & -0.59 & $\cdots$ \\
\hline \multicolumn{17}{|c|}{ Ethinic group (fief = White UK Hentoge) } \\
\hline White European Heritage & $-0,07$ & 0.13 & -0.09 & & 0.05 & 006 & 0.14 & & 0.02 & 0.54 & 0.077 & & -0.24 & 0.12 & -0.33 & - \\
\hline Blad: Garibbean Heritage & 0.15 & 0.16 & 0.18 & & 0.09 & 0.08 & 0.23 & & 0.07 & 0.05 & 0.25 & & -0.30 & 0.14 & -0.42 & - \\
\hline Hack-African Heritage & 0.13 & 0.19 & 0.16 & & 0.01 & 009 & 001 & & 0.12 & 0.06 & 0.45 & & -0.27 & 0.17 & -0.37 & \\
\hline Any Otter Ethic Minority & 0.16 & 0.19 & 0.20 & & 004 & 009 & 0.10 & & 0.03 & 0.06 & 0.11 & & -0.17 & 0.17 & -024 & \\
\hline Indian & 0.39 & 020 & 0.45 & & 0.14 & 0.09 & 0.38 & & 0.02 & 0.07 & 0.08 & & 0.44 & 0.18 & 061 & - \\
\hline Pakistani & 0.83 & 0.16 & 0.04 & & -0.08 & $0 @ 7$ & -0.21 & & -0.08 & 0.05 & -0.31 & & -0.05 & 0.14 & -0.07 & \\
\hline Bangladeshi & -0.43 & 0.26 & -0.52 & & 0.23 & 0.12 & 0.62 & - & 0.06 & 0.08 & 0.21 & & 0.27 & 0.23 & 0.38 & \\
\hline Mued Race & 0.11 & 0.12 & 0.13 & & 000 & 0.05 & 001 & & 0.03 & 0.54 & 0.11 & & -005 & 0.10 & -0.07 & \\
\hline \multicolumn{17}{|c|}{ Number of siblingr at age $3 / 5$ (Ref $=N b$ Siblings) } \\
\hline 1 sibling & 0.02 & 006 & 0.03 & & 0.03 & $a c B$ & 009 & & -0.01 & 0 on & -0.08 & & -002 & 0.06 & -0.03 & \\
\hline 2 siblings & 0.05 & 0.07 & 006 & & 0.01 & 0.03 & 0.02 & & -0.01 & 0.02 & -0.02 & & 0.01 & 0.06 & 0.01 & \\
\hline
\end{tabular}

Table 6. Continued.

\begin{tabular}{|c|c|c|c|c|c|c|c|c|c|c|c|c|c|c|c|c|}
\hline & \multicolumn{4}{|c|}{ Educational computing at age 11} & \multicolumn{4}{|c|}{$\begin{array}{l}\text { Irteractive learning processes at age } \\
11\end{array}$} & \multicolumn{4}{|c|}{ Individual activities at age 11} & \multicolumn{4}{|c|}{ Computer gemes at age 11} \\
\hline & Coeff. & $\begin{array}{l}\text { Std. } \\
\text { Eror }\end{array}$ & $\begin{array}{l}\text { Effect } \\
\text { Size }\end{array}$ & Sig. & Coeff. & $\begin{array}{l}\text { Std. } \\
\text { Error }\end{array}$ & $\begin{array}{l}\text { Effect } \\
\text { Sue }\end{array}$ & Sig. & Coett. & $\begin{array}{l}\text { Std. } \\
\text { Errox }\end{array}$ & $\begin{array}{l}\text { Effed } \\
\text { Size }\end{array}$ & Sig. & Coeff. & $\begin{array}{l}\text { Std. } \\
\text { Enror }\end{array}$ & $\begin{array}{l}\text { Effect } \\
\text { Six }\end{array}$ & Sig \\
\hline 3+ siblings & 0.12 & 0.09 & 0.15 & & 0.11 & 0.04 & 0.30 & $*$ & 0.01 & 0.03 & 0.03 & & 020 & 0.08 & 0.27 & $\cdot$ \\
\hline \multicolumn{17}{|c|}{ Parents' highest SES at age 3/5 (hef = Professional, Non-Manuel) } \\
\hline $\begin{array}{l}\text { Other profeasional non- } \\
\text { manual }\end{array}$ & 0.03 & 009 & 0.04 & & 0.03 & 0.04 & 0.07 & & 0.01 & 0.03 & 0.02 & & -0.05 & 0.08 & -006 & \\
\hline Skilled non-manual & -0.01 & 0.10 & -0.01 & & -0.01 & 0.04 & -0.02 & & 0.00 & 0.03 & 0.01 & & -0.05 & 0.09 & -0.07 & \\
\hline Shilled manual & -0.03 & 0.11 & -0.04 & & -0.02 & 0.05 & -0.05 & & 0.02 & 0.04 & 0.07 & & -0.12 & 0.10 & -0.16 & \\
\hline Semiskilled & -0.19 & 0.12 & -0.23 & & -0.07 & 0.06 & -0.19 & & 0.01 & 0.04 & 0.03 & & 0.02 & 0.11 & 0.03 & \\
\hline Unskilled & -0.58 & 0.22 & -0.69 & $*$ & -0.19 & 0.10 & -0.52 & & -0.14 & 0.07 & -0.49 & & -0.15 & 0.20 & -0.21 & \\
\hline Never worked & -003 & 0.23 & -0.04 & & -0.09 & 0.10 & -0.25 & & -0.06 & 0.07 & -0.21 & & -000 & 0.20 & -000 & \\
\hline \multicolumn{17}{|c|}{ Family salay at oge 7 Ref = No Salary) } \\
\hline $2,500-15,000$ salary & 0.02 & 007 & 0.03 & & 0.04 & 0.03 & 0.09 & & -0.03 & 0.02 & -0.10 & & 0.04 & 0.06 & 006 & \\
\hline f17,500-27,500 salary & 0.07 & 008 & 0.09 & & 0.07 & 0.03 & 0.18 & & 0.02 & 0.02 & 0.07 & & 0.05 & 0.07 & 007 & \\
\hline £3,000-35,000 salary & -0.04 & 009 & -0.04 & & 0.05 & 0.04 & 0.14 & & 0.02 & 0.03 & 0.09 & & -0.03 & 0.08 & -004 & \\
\hline $637,500-66,000$ sala $y$ & 0.11 & 008 & 0.13 & & 0.04 & 0.04 & 0.10 & & 0.02 & 0.03 & 0.08 & & -0.03 & 0.07 & -0.04 & \\
\hline f67,500-132,000 salary & 0.13 & 0.12 & 0.16 & & 0.04 & 0.05 & 0.10 & & 0.02 & 0.04 & 0.07 & & -0.19 & 0.10 & -0.27 & \\
\hline \multicolumn{17}{|c|}{ Mother's highest qualifications level $\alpha$ age 3/5 Ref $=$ None) } \\
\hline Vocational & 0.34 & 0.09 & 0.41 & $\cdots$ & 0.13 & 0.04 & 0.36 & 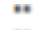 & 0.07 & 0.03 & 0.26 & • & 0.04 & 0.08 & 006 & \\
\hline Academic age 16 & 0.30 & 0.08 & 0.36 & *.* & 0.11 & 0.04 & 0.28 & $*$ & 0.03 & 0.03 & 0.10 & & 0.11 & 0.07 & 0.15 & \\
\hline Academic age 18 & 028 & Q.11 & 0.33 & $*$ & 0.20 & 0.05 & 0.54 & + & 0.09 & 0.03 & 0.33 & $\dot{*}$ & 0.12 & 0.09 & 0.16 & \\
\hline Degree or higher degree & 0.14 & 0.10 & 0.16 & & 0.21 & 0.05 & 0.55 & $\cdots$ & 0.10 & 0.03 & 0.38 & ** & -0.01 & 0.09 & -0.01 & \\
\hline Other peofessional & -002 & 021 & -0.02 & & -0.09 & 0.10 & -0.24 & & 0.02 & 0.07 & 0.07 & & -0.27 & 0.18 & -0.37 & \\
\hline Mother's age & -0.11 & 0.04 & -0.18 & $*$ & -0.07 & 0.02 & -0.24 & 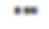 & -0.03 & 0.01 & -0.12 & & -0.10 & 0.04 & -0.18 & $*$ \\
\hline Intercept & 122 & 0.18 & & $\cdots$ & 1.11 & 0.08 & & $m$ & 0.91 & 0.06 & & $\cdots$ & 1.78 & 0.16 & & $\cdots$ \\
\hline Variana Level 2 & 0,00 & 000 & & $\cdots$ & 0.00 & 0.00 & & $m$ & 0.00 & 000 & & $*$ & 0.00 & 0.00 & & \\
\hline Variance Level 1 & 0.69 & 0.03 & & $\cdots$ & 0.14 & 0.01 & & m. & 0.07 & 0.00 & & $\cdots$ & 0.53 & 0.02 & & $\cdots$ \\
\hline Toal Variane & 0.69 & & & & 0.14 & & & & 0.07 & & & & 0.53 & & & \\
\hline Number of Observations & 1,364 & & & & 1,364 & & & & 1,364 & & & & 1,364 & & & \\
\hline Number of Groups & 627 & & & & 627 & & & & 627 & & & & 627 & & & \\
\hline $\begin{array}{l}\text { Deviance }(-2 x \log \\
\text { Restricted Likellhood) }\end{array}$ & 3373.89 & & & & $1,231.10$ & & & & 334.18 & & & & $3,007.60$ & & & \\
\hline Intra-Class Correlation (CC) & 0.000 & 0000 & & & 0.023 & 0.025 & & & 0.000 & 0.000 & & & 0.00 & 0.008 & & \\
\hline $\begin{array}{l}\text { \% Reduction Student } \\
\text { Variance }\end{array}$ & 18.45 & & & & 25.18 & & & & 20.14 & & & & 1861 & & & \\
\hline \% Reduction School Variano & 100.00 & & & & 83.98 & & & & 100.00 & & & & 9929 & & & \\
\hline \%6 Reduction Total Variance & 21.78 & & & & 31.04 & & & & 31.61 & & & & 19.14 & & & \\
\hline
\end{tabular}


Table 7. Contextualised models for lower secondary school measures of HLE at age 14.

\begin{tabular}{|c|c|c|c|c|c|c|c|c|c|c|c|c|c|c|c|c|c|c|c|c|}
\hline & \multicolumn{4}{|c|}{ Enrichment at age 14} & \multicolumn{4}{|c|}{ Interest at age 14} & \multicolumn{4}{|c|}{ Computer at age 14} & \multicolumn{4}{|c|}{ Support at age 14} & \multicolumn{4}{|c|}{ Supervision at age 14} \\
\hline & Coeff. & $\begin{array}{l}\text { Std } \\
\text { Erro }\end{array}$ & $\begin{array}{l}\text { Effed } \\
\text { Size }\end{array}$ & Sig. & Coeff. & $\begin{array}{l}\text { Std } \\
\text { Erra }\end{array}$ & $\begin{array}{l}\text { Effect } \\
\text { Szee }\end{array}$ & $\operatorname{sig}$ & Coeff. & $\begin{array}{l}\text { Stl. } \\
\text { Ema }\end{array}$ & $\begin{array}{l}\text { Effed } \\
\text { Size }\end{array}$ & $\operatorname{sig}$ & Coeff. & $\begin{array}{l}\text { Std. } \\
\text { Erro }\end{array}$ & $\begin{array}{c}\text { Effed } \\
\text { Saze }\end{array}$ & Sig & Coeff. & $\begin{array}{l}\text { Std } \\
\text { Enra }\end{array}$ & $\begin{array}{l}\text { Effed } \\
\text { Size }\end{array}$ & Sig \\
\hline \multicolumn{21}{|c|}{ Eariy Years HIE (Ref $=0-13)$} \\
\hline 14-19 & 0.14 & 0.19 & 0.10 & & -0.02 & 0.17 & -0.01 & & 0.88 & 0.38 & 0.31 & - & -0.08 & 0.27 & -0.04 & & -0.06 & 0.15 & -0.06 & \\
\hline $20-24$ & 0.27 & 0.19 & 0.19 & & 0.25 & 0.17 & 0.20 & & 0.75 & 0.39 & 0.26 & & -0.22 & 0.28 & -0.11 & & -0.13 & 0.15 & -0.12 & \\
\hline $25-32$ & 0.21 & 0.19 & 0.15 & & 0.27 & 0.17 & 0.22 & & 1.10 & 0.38 & 0.38 & $\ddot{*}$ & -0.04 & 0.27 & -0.02 & & -0.22 & 0.15 & -0.20 & \\
\hline$>33$ & 0.49 & 0.21 & 0.35 & - & 0.38 & 0.18 & 0.31 & - & 1.37 & 0.2 & 0.47 & $*$ & 0.30 & 0.30 & 0.15 & & -0.20 & 0.16 & -0.18 & \\
\hline \multicolumn{21}{|c|}{ HE Home Computing age 7 Pef $=$ Law) } \\
\hline $\begin{array}{l}\text { HLE Home Computing } \\
\text { Medium }\end{array}$ & -0.10 & 0.11 & -0.07 & & 0.05 & 0.10 & 0.04 & & 0.34 & 0.22 & 0.12 & & Q.40 & 0.16 & 0.20 & $\cdot$ & 0.01 & 0.09 & 0.01 & \\
\hline $\begin{array}{l}\text { Hif Home Computing } \\
\text { High }\end{array}$ & 0.15 & 0.14 & 0.10 & & 0.07 & 0.12 & 0.06 & & 0.37 & 0.29 & 0.13 & & 0.69 & 0.20 & 0.35 & $\cdots$ & 0.11 & 0.11 & 0.10 & \\
\hline \multicolumn{21}{|c|}{ Hef interaction age 7 (Ref $=$ Law) } \\
\hline $\begin{array}{l}\text { HLE Irteraction } \\
\text { Medium }\end{array}$ & 0.16 & 0.13 & 0.11 & & 0.40 & 0.11 & 0.33 & $\cdots$ & -0.05 & 0.25 & -0.02 & & 0.43 & 0.18 & 0.22 & $\cdot$ & 0.31 & 0.10 & 0.28 & $*$ \\
\hline \multicolumn{20}{|c|}{ HeE Enrictiment Outing age 7 (hef $=$ Low) } & $\cdots$ \\
\hline $\begin{array}{l}\text { HLE Enrichment } \\
\text { Outings Medium }\end{array}$ & 0.33 & 0.16 & 0.23 & $\cdot$ & -0.10 & 0.14 & -0.08 & & 0.24 & 0.32 & 0.08 & & 0.64 & 0.22 & 0.33 & $*$ & 0.03 & 0.12 & 0.03 & \\
\hline $\begin{array}{l}\text { HLE Enrichment } \\
\text { Outings High } \\
\text { HE Expressive Play age }\end{array}$ & $\begin{array}{r}0.40 \\
7(\text { Ref }=L\end{array}$ & 0.20 & 0.28 & $\cdot$ & -0.03 & 0.18 & -0.02 & & 0.23 & 0.41 & 0.08 & & 1.00 & 0.29 & 0.51 & $\cdots$ & 0.16 & 0.15 & 0.14 & \\
\hline HLE Fay Medium & -0.03 & 0.13 & -0.02 & & 0.13 & 0.11 & 0.11 & & 0.51 & 0.26 & 0.18 & & -0.04 & 0.17 & -0.02 & & -0.08 & 0.10 & -0.07 & \\
\hline HLE Flay Hgh & -0.02 & 0.17 & -0.01 & & 0.11 & 0.14 & 0.09 & & 0.22 & 0.34 & 0.08 & & 0.18 & 0.23 & 0.09 & & 0.06 & 0.13 & 0.05 & \\
\hline \multicolumn{21}{|c|}{ HLE Educational Computing age 11 (Nef = Low) } \\
\hline $\begin{array}{l}\text { HLE Educatonal } \\
\text { Computing Medum }\end{array}$ & 0.04 & 0.14 & 0.03 & & -0.06 & 0.12 & -0.05 & & 0.30 & 0.29 & 0.10 & & 0.30 & 0.19 & 0.15 & & -0.06 & 0.11 & -0.06 & \\
\hline $\begin{array}{l}\text { HLE Eduatonal } \\
\text { Computing high }\end{array}$ & -0.49 & 0.20 & -0.35 & $\cdot$ & -0.10 & 0.17 & -0.08 & & 1.26 & 0.41 & 0.44 & $*$ & 0.34 & 0.28 & 0.17 & & -0.03 & 0.15 & -0.03 & \\
\hline \multicolumn{21}{|c|}{ HLE Learning age $n$ (1) $=$ Law) } \\
\hline HLE Learning Medium & 0.02 & 0.16 & 0.01 & & 0.37 & 0.13 & 0.30 & ** & -0.19 & 0.31 & -0.06 & & 0.40 & 021 & 0.20 & & 0.31 & 0.12 & 0.29 & * \\
\hline HLE Learning High & 0.18 & 0.21 & 0.13 & & 0.44 & 0.18 & 0.36 & • & -0.51 & 0.2 .2 & -0.18 & & 1.09 & 029 & $0.5 s$ & $\cdots$ & 0.50 & 0.16 & 0.45 & ** \\
\hline \multicolumn{21}{|c|}{ HLE Indvidual adtivities age 11 Ref = Low) } \\
\hline \multirow{2}{*}{$\begin{array}{l}\text { HLE Individual } \\
\text { Activities Medium }\end{array}$} & $\cos$ & 0.14 & 0.94 & & -0.14 & 0.12 & -0.11 & & -0.01 & 0.28 & -0.00 & & -0.26 & 0.19 & -0.13 & & -0.10 & 0.11 & -0.09 & \\
\hline & 0.27 & 0.19 & 0.19 & & -0.14 & 0.17 & -0.12 & & -0.30 & 0.38 & -0.10 & & -0.24 & 0.27 & -0.12 & & -0.22 & 0.14 & -0.20 & \\
\hline
\end{tabular}

(Continued)

Table 7. Continued.

\begin{tabular}{|c|c|c|c|c|c|c|c|c|c|c|c|c|c|c|c|c|c|c|c|c|}
\hline & \multicolumn{4}{|c|}{ Enrichmert at age 14} & \multicolumn{4}{|c|}{ Interest at age 14} & \multicolumn{4}{|c|}{ Computer at age 14} & \multicolumn{4}{|c|}{ Support at age 14} & \multicolumn{4}{|c|}{ Supervision at age 14} \\
\hline & Coeff. & $\begin{array}{l}\text { Std } \\
\text { Erro }\end{array}$ & $\begin{array}{l}\text { Effed } \\
\text { Sare }\end{array}$ & Sig. & Coeff. & $\begin{array}{l}\text { Std } \\
\text { Enro }\end{array}$ & $\begin{array}{l}\text { Effed } \\
\text { Seze }\end{array}$ & $\operatorname{sig}$ & Coeff. & $\begin{array}{l}\text { Stid. } \\
\text { Error }\end{array}$ & $\begin{array}{l}\text { Effed } \\
\text { Size }\end{array}$ & $\operatorname{sig}$ & Coeff. & $\begin{array}{l}\text { Stl. } \\
\text { Ema }\end{array}$ & $\begin{array}{c}\text { Effed } \\
\text { Slae }\end{array}$ & $\operatorname{sig}$ & Coeff. & $\begin{array}{l}\text { Std } \\
\text { Enor }\end{array}$ & $\begin{array}{l}\text { Effed } \\
\text { Size }\end{array}$ & Sig. \\
\hline $\begin{array}{l}\text { HEF Individual } \\
\text { Activities High }\end{array}$ & 11 (Ref: & Low) & & & & & & & & & & & & & & & & & & \\
\hline $\begin{array}{l}\text { HE Computer Games } \\
\text { Medium }\end{array}$ & -0.11 & 0.12 & -0.08 & & -0.24 & 0.10 & -0.19 & $\cdot$ & 0.16 & 0.24 & 0.05 & & -0.12 & 0.16 & -0.06 & & -0.07 & 0.09 & -0.07 & \\
\hline $\begin{array}{l}\text { HE Computer Games } \\
\text { High }\end{array}$ & -0.00 & 0.17 & -0.00 & & -0.16 & 0.15 & -0.13 & & 0.12 & 0.35 & 0.04 & & -0.29 & 0.23 & -0.15 & & 0.07 & 0.13 & 0.07 & \\
\hline $\begin{array}{l}\text { Girls or Gender } \\
\text { Ref=Eoys) }\end{array}$ & 0.33 & 0.09 & 0.23 & $\cdots$ & -0.09 & 0.08 & -0.07 & & 0.88 & 0.18 & 0.30 & $\cdots$ & -0.07 & 0.12 & -0.04 & & 0.10 & 0.00 & 0.09 & \\
\hline \multicolumn{21}{|c|}{ Ethinic group (Ref = White UK Hertage) } \\
\hline $\begin{array}{l}\text { White European } \\
\text { Heritage }\end{array}$ & 0.20 & 0.23 & Q.14 & & -0.07 & 0.21 & -0.05 & & 1.26 & 0.46 & 0.44 & $*$ & -0.12 & 0.34 & -0.06 & & 0.02 & 0.17 & 0.02 & \\
\hline $\begin{array}{l}\text { Flad Caribbean } \\
\text { Heritage }\end{array}$ & 0.23 & 0.28 & 0.16 & & -0.18 & 0.25 & -0.15 & & 0.35 & 0.55 & 0.12 & & 0.46 & 0.39 & 0.23 & & 0.16 & 0.20 & 0.15 & \\
\hline Black African Heritage & 0.76 & 0.42 & 0.54 & & 0.30 & 0.35 & 0.24 & & -0.42 & 0.81 & -0.15 & & 1.37 & 0.57 & 0.70 & * & 0.86 & 0.31 & 0.79 & $*$ \\
\hline $\begin{array}{l}\text { Any Other Ethric } \\
\text { Minoity }\end{array}$ & 0.73 & 0.34 & 0.52 & * & 0.26 & 0.33 & 0.21 & & 0.67 & $0 . \pi$ & 0.23 & & 0.59 & 0.53 & 0.30 & & -0.12 & 0.20 & -0.11 & \\
\hline Indian & 0.45 & 0.20 & 0.32 & & 0.10 & 0.26 & 0.08 & & -0.46 & 0.60 & -0.16 & & 1.29 & 0.41 & 0.66 & $*$ & 0.14 & $0.2 n$ & 0.12 & \\
\hline Pakistani & 0.88 & 0.24 & 0.62 & $\cdots$ & -0.00 & 0.23 & -0.00 & & -0.67 & 0.51 & -0.23 & & 0.97 & 0.36 & 0.50 & $*$ & 0.21 & 0.19 & 0.19 & \\
\hline Bangladeshi & 0.60 & 0.56 & 0.42 & & -0.65 & 0.52 & -0.53 & & -1.14 & 1.13 & -0.40 & & -0.20 & $0 . \pi$ & -0.10 & & -0.45 & 0.43 & -0.41 & \\
\hline Mixed Race & 0.28 & 0.20 & 0.20 & & 0.01 & 0.17 & 0.00 & & 0.58 & 0.41 & 0.20 & & 0.37 & $0.2 y$ & 0.19 & & -0.02 & 0.15 & -0.02 & \\
\hline \multicolumn{21}{|c|}{ Number of siblings at age $3 / 5$ (Rief = No Siblings) } \\
\hline 1 sibling & -0.05 & 0.11 & -0.04 & & -0.09 & 0.10 & -0.07 & & 0.00 & 0.22 & 0.00 & & 0.01 & 0.15 & 0.01 & & 0.04 & 0.08 & 0.04 & \\
\hline 2 siblings & -0.15 & 0.12 & -0.11 & & -0.17 & 0.10 & -0.14 & & 0.35 & 0.24 & 0.12 & & -0.15 & 0.16 & -0.08 & & -0.08 & 0.09 & -0.07 & \\
\hline $3+$ siblings & -0.23 & 0.15 & -0.16 & & -0.27 & 0.14 & -0.22 & * & 0.21 & 0.31 & 0.07 & & -0.32 & 0.21 & -0.16 & & -0.15 & 0.12 & -0.14 & \\
\hline \multicolumn{21}{|c|}{ Parents' highest SES of age 3/5 (Aef = Professionel, Mbr-Manual) } \\
\hline $\begin{array}{l}\text { Other professional } \\
\text { non-manual }\end{array}$ & -0.50 & 0.14 & -0.35 & $\cdots$ & 0.06 & 0.12 & 0.05 & & 0.06 & 0.28 & 0.02 & & 0.05 & 0.19 & 0.02 & & -0.15 & 0.11 & -0.14 & \\
\hline Skilled non-manual & -0.57 & 0.16 & -0.40 & w. & -0.05 & 0.14 & -0.04 & & 0.11 & 0.33 & 0.04 & & -0.17 & 0.22 & -0.09 & & 0.02 & 0.12 & 0.01 & \\
\hline Sceilled manual & -0.66 & 0.19 & -0.46 & ** & -0.00 & 0.17 & -0.00 & & -0.14 & 0.39 & -0.05 & & -0.05 & $0.2 z$ & -0.03 & & -0.01 & 0.15 & -0.01 & \\
\hline Semiskilled & -0.77 & 0.21 & -0.55 & *** & -0.19 & 0.18 & -0.15 & & -0.22 & 0.42 & -0.08 & & -0.17 & 0.28 & -0.08 & & -0.15 & 0.16 & -0.14 & \\
\hline Unskilled & -0.44 & 0.41 & -0.31 & & -0.93 & 0.37 & -0.76 & - & -1.37 & 0.87 & -0.48 & & -0.25 & 061 & -0.13 & & -0.17 & 0.32 & -0.16 & \\
\hline Never worked & -0.82 & 0.41 & -0.58 & * & -0.05 & 0.42 & -0.04 & & 1.82 & 0.84 & 0.63 & $\cdot$ & 0.14 & 070 & 0.07 & & -0.95 & 032 & -0.87 & $*$ \\
\hline \multicolumn{21}{|c|}{ Family salary at age 7 (Ref $=$ No Salay) } \\
\hline $62,500-15,000$ salary & -0.09 & 0.14 & -0.06 & & -0.16 & 0.12 & -0.13 & & 0.22 & 0.28 & 0.08 & & -0.08 & 0.19 & -0.04 & & -0.08 & 0.11 & -0.07 & \\
\hline f17,500-27,500 salary & -0.18 & 0.14 & -0.13 & & -0.03 & 0.12 & -0.03 & & -0.01 & 0.28 & -0.00 & & 0.11 & 0.19 & 0.05 & & 0.03 & 0.10 & 0.03 & \\
\hline
\end{tabular}




\begin{tabular}{|c|c|c|c|c|c|c|c|c|c|c|c|c|c|c|c|c|c|c|c|c|}
\hline$£ 30,000-35,000$ salary & -0.18 & 0.15 & -0.13 & & 0.00 & 0.13 & 0.00 & & 0.46 & 0.31 & 0.16 & & 0.14 & 0.22 & 0.07 & & 0.00 & 0.12 & 0.00 & \\
\hline E37,500-66,000 salary & -0.01 & 0.14 & -0.01 & & 0.03 & 0.13 & 0.02 & & 0.46 & 0.29 & 0.16 & & 0.24 & 0.20 & 0.12 & & -0.08 & 0.11 & $-0,07$ & \\
\hline 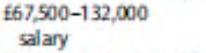 & 0.02 & 0.19 & 0.01 & & -0.02 & 0.17 & -0.01 & & 0.14 & 0.39 & 0.05 & & 0.49 & 0.26 & 0.25 & & -0.00 & 0.15 & -0.00 & \\
\hline \multicolumn{21}{|c|}{ Mother's highest qualifications level at age 3/5 (Ref $=$ Nbne) } \\
\hline Vocational & -0.06 & 0.16 & -0.05 & & 0.36 & 0.14 & 0.29 & - & 0.23 & 0.34 & 0.08 & & 0.38 & 0.23 & 0.19 & & -0.06 & 0.13 & -0.06 & \\
\hline Academic age 16 & -0.02 & 0.14 & -0.02 & & 0.35 & 0.12 & 0.29 & $*$ & 0.22 & 0.29 & 0.08 & & 0.29 & 0.20 & 0.15 & & -0.02 & 0.11 & $-0,02$ & \\
\hline Academic age 18 & 0.54 & 0.19 & 0.38 & 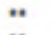 & 0.31 & 0.17 & 0.25 & & -0.60 & 0.39 & -0.21 & & 0.27 & 0.27 & 0.14 & & 0.12 & 0.15 & 0.11 & \\
\hline $\begin{array}{l}\text { Degree or higher } \\
\text { degree }\end{array}$ & 0.55 & 0.18 & 0.38 & $*$ & 0.23 & 0.16 & 0.19 & & -0.46 & 0.37 & -0.16 & & -032 & 0.26 & -0.16 & & -0.12 & 0.14 & -0.11 & \\
\hline Other professional & 0.15 & 0.32 & 0.11 & & 0.33 & 0.28 & 0.27 & & 0.05 & 0.66 & 0.02 & & 0.14 & 0.45 & 0.07 & & 0.31 & 0.25 & 0.28 & \\
\hline Mother's age & 0.07 & 0.07 & 0.06 & & 0.02 & 0.06 & 0.02 & & -0.06 & 0.15 & -0.03 & & -0.03 & 0.10 & -0.02 & & 0.03 & 0.06 & 0.03 & \\
\hline Intercept & 1.69 & 0.32 & & $\cdots$ & 6.57 & 0.29 & & $\cdots$ & 5.21 & 0.67 & & $\cdots$ & 272 & 0.46 & & $\cdots$ & 4.91 & 0.25 & & $\cdots$ \\
\hline Variance Level 2 & 0.02 & 0.04 & & & 003 & 0.03 & & $\cdots$ & 0.10 & 0.13 & & & 003 & 007 & & & 002 & 0.02 & & $\cdots$ \\
\hline Varlance Level 1 & 201 & 0.09 & & $\cdots$ & 151 & 0.06 & & $\cdots$ & 8.30 & 0.35 & & $\cdots$ & 3.84 & 0.17 & & $\cdots$ & 1.19 & 0.05 & & $\cdots$ \\
\hline Total Variance & 203 & & & & 1.54 & & & & 8.40 & & & & 3.87 & & & & 1.21 & & & \\
\hline $\begin{array}{l}\text { Number of } \\
\text { Observations }\end{array}$ & 1,325 & & & & 1,316 & & & & 1,317 & & & & 1,295 & & & & 1,342 & & & \\
\hline Number of Groups & 435 & & & & 444 & & & & 435 & & & & 443 & & & & 440 & & & \\
\hline $\begin{array}{l}\text { Deviance (-2x Log } \\
\text { Plestricted } \\
\text { Likel ihood) }\end{array}$ & $4,697.15$ & & & & 4,30447 & & & & $6,539.42$ & & & & $5,426.73$ & & & & 4,06283 & & & \\
\hline $\begin{array}{l}\text { Intra-Class Correlation } \\
\text { ACC }\end{array}$ & 0,009 & 0.019 & & & 0.020 & 0.018 & & & 0.012 & 0.016 & & & 0.008 & 0.019 & & & 0.016 & 0.017 & & \\
\hline $\begin{array}{l}\text { \% Feduction Student } \\
\text { Variance }\end{array}$ & 9.07 & & & & 1287 & & & & 11.86 & & & & 11.19 & & & & 932 & & & \\
\hline $\begin{array}{l}\text { \% Peduction School } \\
\text { Variance }\end{array}$ & 93.43 & & & & 24.77 & & & & 37.63 & & & & 68.84 & & & & -186.26 & & & \\
\hline $\begin{array}{l}\text { \% Reduction Total } \\
\text { Variance }\end{array}$ & 18.03 & & & & 13.14 & & & & 1230 & & & & 1247 & & & & 8.33 & & & \\
\hline
\end{tabular}

\section{HLE measures at age 11 as predictors of HLE measures at age 14}

Parents who joined with their children in sport activities, games, or in visiting a museum at age 11 tended to be more likely to report that they helped them with difficult homework, using the internet or with downloading educational software at age 14 ( $E S=0.55$, see Table 7). According to their own reports, these parents also showed significantly more interest in their children's school experiences by talking to them about school work and General Certificate of Secondary Education (GCSE) subjects ( $E S=0.36$ ). Their children experienced higher levels of parent supervision of learning at home at age 14 as these parents reported they checked homework was completed $(E S=0.45)$. Children who frequently used the computer at age 11 were significantly more likely to use it at age 14 as well, but interestingly they were significantly less likely to report they read for pleasure or went on educational visits with family. This was found even when the effect of child gender was controlled in the models (gender being associated with different patterns of reported computer use).

\section{Discussion}

This paper makes a new contribution to the existing literature on home learning environments by showing how various measures of the children's and adolescents' HLE collected at different ages are associated over time when their individual and family background characteristics were controlled. It also focuses on multiple dimensions of HLE based on parent reports at different ages, covering a wide range of parent-child interactive activities (reading and playing together), parent-child shared activities (using the computer or participating in sport), individual children activities (dance, painting, reading), computer use, learning and playing activities, but also parental learning support and supervision in adolescence. At age 14, data from questionnaire reports from both parents and adolescents themselves were included. The paper provides evidence of the reliability and robustness of these new measures at different ages and evidence of predictive validity over time through follow up of the same sample across pre-school, primary, and lower secondary phases of education. These measures provide new and broader insight into the types of home learning 
activities parents and children engaged in at different ages in England during the first decade or so of the new millennium. In line with previous studies, parents who were providing a stimulating and rich HLE in the early years were also more likely to continue to provide stimulating home learning environments when children were older (Sy, Gottfried, \& Gottfried, 2013). This was evident in terms of correlational associations but also when relevant family background characteristics were controlled for in the multilevel models. As the Bronfenbrenner ecological model (Bronfenbrenner,1994) suggests, the effects of early years HLE were stronger on HLE dimensions measured closer in time (at age 7 and 11, a proximal effect), and weaker effects were found for HLE dimensions measured later in time (in adolescence, a more distal effect). Also, the strength of the relationships depended on the nature/content/ type of the activities, similar (proximal) activities being more strongly associated over time than different ones. Thus, especially strong effects were found for the early years HLE and parent-child one-to-one interactions at age 7 and 11. Moreover, strong effects were also found for enrichment activities at age 7 when predicting interactive learning activities at age 11 but also between individual activities like expressive play at age 7 and dancing or painting at age 11.

Parental engagement in interactive learning activities at age 11 significantly predicted their involvement and support of their child's learning at age 14. This may also reflect parents' perceptions of changes in children's needs, where at later ages the parental educational involvement is less active in terms of school visits or interactions with the teachers, but more supportive and encouraging of adolescents' autonomy and independence (Kaplan Toren, 2013). Computer-related activities measured at different ages showed distinct patterns of association. The situation may be complicated by the growth in different types of IT activities and increases in parents' own skills as well as changes in children's skills and interests as they developed. The project did not measure the use of tablets or smart phones as they were not common at younger ages, but clearly these are now a major part of even young children's lives a decade later. Thus, the historical context should be borne in mind in interpreting these findings.

When considering the findings, it should be noted that the various background predictors showed strong associations with the early years HLE measure in this study. Thus, the early years HLE may "absorb" some of the effects of most family background characteristics in the multilevel models that investigate the HLE at later ages. Nonetheless, it is interesting to note that some of the background characteristics remained significant predictors of later HLE, even when the effect of the early years HLE was controlled. Thus, SES was still significantly related to enrichment activities at age 7 and academic enrichment at age 14, with more disadvantaged students making fewer visits to libraries and museums during lower primary school and secondary school. This supports the findings of national and longitudinal studies conducted in the US that indicate that poor children were less likely to visit libraries and museums, go on educational trips, or attend theatrical performances (Bradley \& Corwyn, 2002; Bradley et al., 2001; Brooks-Gunnetal.,1995). The findings, however, also throw more light on how low SES may increase the risk of poor educational outcomes and compound the effects of socioeconomic disadvantage by shaping home learning experiences and parental activities with children at different ages. For example, the results show that parents with low SES were less likely to engage in learning activities with their child at younger ages or in school-related activities like supervising/helping with homework later in secondary school. Such parents may feel less confident about getting involved with the school activities 
because they may lack the knowledge about the school system, or because of their own poor educational experiences (Lee \& Bowen, 2006). On the other hand, parents with a high SES may be more aware of the importance of education for success at school and employment prospects in future, and this may result in different ways of supporting their children at different ages and stages of schooling (Davis-Kean, 2005; Hartas, 2011; Jeynes, 2007). This reveals the importance of studying change and stability in children's HLE experiences from pre-school age into adolescence (Sammons, Toth, Sylva, Melhuish, etal., 2015) and addressing different features of HLE experiences in different phases of education. Elsewhere, we have shown how the HLE predicts differences in children's attainment and academic progress as well as other important learning-related behaviours such as selfregulation (Sammons, Toth, \& Sylva, 2015; Sammons, Toth, Sylva, Melhuish,et al.,2015). The differences in HLE experiences over time are associated with background characteristics and with educational outcomes, thus being an important feature to study further to understand the long standing patterns of equity differences in outcomes evident in England, but also in many other countries (Sammons, Toth, \& Sylva, 2018).

Various measures of disadvantage seem to be stronger predictors of the early years HLE pointing to the potential of early intervention work with parents to address the equity gap (Sammons, 2010). At later ages, it may be more important to differentiate support (tailored programmes) to address different aspects of children or young people's HLE experiences. Further research would be of value to explore the role of background characteristics in shaping the HLE at different ages in various country contexts and to establish whether different forms of HLE support (in school or working with parents) may be more effective in narrowing the equity gap in learning experiences in and out of home as well as the attainment gap.

Longitudinal research that follows up children and families across different phases of education, such as the EPPSE study in England, can provide further insights into the role of home learning environments and how experiences at home play a part in shaping educational outcomes. Elsewhere, we have discussed findings on the relationships between children's academic outcomes at different ages and their specific HLE experiences based on the various measures used in this paper (Sammons et al., 2014; Sammons, Toth, \& Sylva, 2015; Sammons, Toth, Sylva, Melhuish, et al., 2015). Here, we have explored in more detail the ways family characteristics and children's HLE experiences are also associated and similarities and differences in these patterns of association at different child ages from early years to primary into secondary schooling. In order to reduce the consequences of social disadvantage and address the large equity gap in educational outcomes evident in many systems, it is important to recognise the way home learning experiences are related to other measures of family disadvantage and how these may interact or compound the effects of socioeconomic disadvantage typically measured by indicators such as family salary, SES, or parents' education levels treated in isolation. There are implications for policies that promote equity and combat the adverse impacts of disadvantage via early intervention and parenting programmes. These were designed to help parents support their children's learning at home and in school (Higgins \& Katsipataki, 2015; Sylva, 2013).

\section{Limitations}

The original EPPSE sample was drawn from a stratified random sample of pre-schools with a "home" group recruited at school entry. It is thus in contrast to birth cohort studies, not a 
random national sample, and the results cannot be generalized. Nonetheless, the characteristics of the EPPSE sample were found to be broadly representative of the national picture for children at different ages in schools in England in terms of characteristics in the Department for Education National Pupil Data base, for example (Sammons et al., 2018; Sylva et al., 2010). The measures of the parent-child activities are based on parent selfreport at ages 3, 7, and 11 and a combination of parent and adolescent self-reports atage 14 . Thus, there is the danger of recall bias and social desirability. Even so, the measures do demonstrate internal validity and predictive validity. Other research approaches such as personal digital devices for parents to record engagement in activities with their children or observations might provide different insights and enable alternative measures of the HLE to be developed. Although the sample is fairly large and includes rich data to explore the associations and effects of a range of background characteristics as predictors of the various HLE measures at different ages, it is linked to a particular context and time frame (England in the early $21^{\text {st }}$ millennium). The rapid expansion of new developments in IT means that the HLE measures related to home computing may be dated now and of limited replicability. The children were in pre-school and primary school before smart phones and tablets were as widespread as it is now the case when they have become a major feature of children's learning activities at home and school and a vital communication tool in terms of social media. New research is needed to explore the way home learning activities and environments are related to digital technologies and their associations with other measures of HLE at different ages and how these may shape children's learning experiences at home and help to influence their educational outcomes.

\section{Notes}

1. Family SES was calculated by considering the highest SES status of the mother or the father in their current occupation at entry to the study.

2. Based on data collected by parent questionnaires completed when students were in KeyStage 1 (ages 5-6 years) of primary school.

3. These continuous measures were used as HLE outcomes (dependent variables) in the multilevel models.

4. These categorical versions of the HLE measures were used as predictors of later HLE (dependent variables) in the multilevel models.

5. Effect sizes (ES) provide a measure of the strength of the relationships between different predictors and the outcomes under study. For more details on ES, see Elliot and Sammons (2004).

Funding This study was part of a research project (EPPSE3-16) funded by the Department of Education, UK.

\section{Notes on contributors}

Dr Katalin Toth was at the time of the study a Research Officer at the University of Oxford. Pam Sammons is Professor of Education at the University of Oxford and Senior Research Fellow at Jesus College, Oxford. Kathy Sylva is Professor of Educational Psychology at the University of Oxford. She is also affiliated with Jesus College. Edward Melhuish is Professor 
of Human Development at the University of Oxford. Iram Siraj is Professor of Child Development \& Education at the University of Oxford. Brenda Taggart was a Senior Research Officer at the UCL Institute of Education, London.

\section{References}

Anders,Y.,Rossbach,H.-G.,Weinert,S.,Ebert,S.,Kuger,S.,Lehrl,S.,\&vonMaurice,J.(2012).Homeand preschool learning environments and their relations to the development of early numeracy skills. Early Childhood Research Quarterly, 27(2), 231-244. doi:10.1016/j.ecresq.2011.08.003

Bradley R. H. (1994). The HOME Inventory: Review and reflections. In H. Reese (Ed.), Advances in child development and behavior (Vol. 25, pp. 241-288). San Diego, CA: Academic.

Bradley, R. H., \& Caldwell, B. M. (1982). The consistency of the home environment and its relation to child development. International Journal of Behavioral Development, 5(4), 445-465. doi:10.1177/ 016502548200500404

Bradley,R.H., Caldwell,B. M.,\&Rock,S. L.(1988).Home environment and school performance: A ten year follow-up and examination of three models of environmental action. Child Development, 59(4), 852-867. doi:10.2307/1130253

Bradley, R. H., \& Corwyn, R. F. (2001). Home environment and behavioral development during early adolescence: The mediating and moderating roles of self-efficacy beliefs. Merrill-Palmer Quarterly, $47(2), 165-187$.

Bradley, R. H., \& Corwyn, R. F. (2002). Socioeconomic status and child development. Annual Review of Psychology, 53, 371-399. doi:10.1146/annurev.psych.53.100901.135233

Bradley, R. H., Corwyn, R. F., Burchinal, M., McAdoo, H. P., \& García Coll, C. (2001). The home environments of children in the United States Part II: Relations with behavioral development through age thirteen. Child Development, 72(6), 1868-1886. doi:10.1111/1467-8624.t01-1-00383

Bradley, R. H., Corwyn, R. F., Caldwell, B. M., Whiteside-Mansell, L., Wasserman, G. A., \& Mink, I. T. (2000). Measuring the home environments of children in early adolescence. Journal of Research on Adolescence, 10(3), 247-288. doi:10.1207/SJRA1003_1 Bronfenbrenner, U. (1994). Ecological models of human development. In T. Husèn \& T. N. Postlethwaite (Eds.), International encyclopedia of education (2nd ed., Vol. 3, pp. 1637-1643). Oxford, UK: Elsevier.

Brooks-Gunn, J., Klebanov, P. K., \& Liaw, F.-R. (1995). The learning, physical, and emotional environment of the home in the context of poverty: The infant health and development program.Children and Youth Services Review, 17(1-2), 251-276. doi:10.1016/0190-7409(95)00011-Z

Burgess,S.R.,Hecht,S.A.,\&Lonigan,C.J.(2002). Relationsofthehomeliteracyenvironment(HLE)to the development of reading-related abilities: A one-year longitudinal study. Reading Research Quarterly, 37(4), 408-426. doi:10.1598/RRQ.37.4.4

Carneiro, P., Meghir, C., \& Parey, M. (2013). Maternal education, home environments, and the development of children and adolescents. Journal of the European Economic Association, 11(s1), 123-160. doi:10.1111/j.1542-4774.2012.01096.x

Davis-Kean, P. E. (2005). The influence of parent education and family income on child achievement: The indirect role of parental expectations and the home environment. Journal of Family Psychology, 19(2), 294-304. doi:10.1037/0893-3200.19.2.294 
Elliot, K., \& Sammons, P. (2004). Exploring the use of effect sizes to evaluate the impact of different influences on child outcomes: Possibilities and limitation. In I. Schagen \& K. Elliot (Eds.), What does it mean? The use of effect sizes in educational research (pp. 6-24). Slough: National Foundation for Educational Research.

Foster, M. A., Lambert, R., Abbott-Shim, M., McCarty, F., \& Franze, S. (2005). A model of home learning environmentandsocialriskfactorsinrelationtochildren'semergentliteracyandsocialoutcomes. Early Childhood Research Quarterly, 20(1), 13-36. doi:10.1016/j.ecresq.2005.01.006

Hartas, D. (2011). Families' social backgrounds matter: Socio-economic factors, home learning and young children's language, literacy and social outcomes. British Educational Research Journal, 37(6), 893-914. doi:10.1080/01411926.2010.506945

Hayes, N., Berthelsen, D. C., Nicholson, J. M., \& Walker, S. (2018). Trajectories of parental involvement in home learning activities across the early years: Associations with socio-demographic characteristics and children's learning outcomes. Early Child Development and Care, 188(10), 14051418. doi:10.1080/03004430.2016.1262362

Higgins, S., \& Katsipataki, M.(2015). Evidence from meta-analysis about parental involvement in education which supports their children's learning. Journal of Children's Services, 10(3), 280-290. doi:10.1108/JCS-02-2015-0009

Jeynes, W. H. (2007). The relationship between parental involvement and urban secondary school student academic achievement: A meta-analysis. Urban Education, 42(1), 82-110. doi:10.1177/ 0042085906293818

Kaplan Toren, N. (2013). Multiple dimensions of parental involvement and its links to young adolescent self-evaluation and academic achievement. Psychology in the Schools, 50(6), 634-649. doi:10. 1002/pits.21698

Kluczniok, K., Lehrl, S., Kuger, S., \& Rossbach, H.-G. (2013). Quality of the home learning environment during preschool age - Domains and contextual conditions. European Early Childhood Education Research Journal, 21(3), 420-438. doi:10.1080/1350293X.2013.814356

Lee, J.-S., \& Bowen, N. K. (2006). Parent involvement, cultural capital, and the achievement gap among elementary school children. American Educational Research Journal, 43(2), 193-218. doi:10.3102/00028312043002193

Leseman, P. P. M., \& De Jong, P. F. (1998). Home literacy: Opportunity, instruction, cooperation and social-emotional quality predicting early reading achievement. Reading Research Quarterly, 33(3), 294-318.

Melhuish, E. C. (2010). Impact of the home learning environment on child cognitive development: Secondary analysis of data from "Growing Up in Scotland". Edinburgh: The Scottish Government.

Melhuish, E. C., Phan, M. B., Sylva, K., Sammons, P., Siraj-Blatchford, I., \& Taggart, B. (2008). Effects of the home learning environment and preschool center experience upon literacy and numeracy development in early primary school. Journal of Social Issues, 64(1), 95-114. doi:10.1111/j.15404560.2008.00550.x

Raikes,H., Pan,B.A., Luze,G.,Tamis-LeMonda,C.S.,Brooks-Gunn,J., Constantine,J. ,...Rodriguez,E.T., (2006). Mother-child book reading in low-income families: Correlates and outcomes during the first three years of life. Child Development, 77(4), 924-953. doi:10.1111/j.1467-8624.2006.00911.xl 
Rodriguez,E.T.,\& Tamis-LeMonda,C.S.(2011).Trajectories of the home learning environment across the first 5 years: Associations with children's vocabulary and literacy skills at pre kindergarten.Child Development, 82(4), 1058-1075. doi:10.1111/j.1467-8624.2011.01614.x

Rodriguez, E. T., Tamis-LeMonda, C. S., Spellmann, M. E., Pan, B. A., Raikes, H., Lugo-Gil, J., \& Luze, G. (2009). The formative role of home literacy experiences across the first three years of life in children from low-income families. Journal of Applied Developmental Psychology, 30(6), 677-694. doi:10.1016/j.appdev.2009.01.003

Rose, E., Lehrl, S., Ebert,S., \& Weinert, S.(2018).Long-term relations between children's language, the home literacy environment, and socioemotional development from ages 3 to 8 . Early Education and Development, 29(3), 342-356. doi:10.1080/10409289.2017.1409096

Sammons, P. (2010). Equity and educational effectiveness. In P. Peterson, E. Baker, \& B. McGaw (Eds.), International encyclopedia of education (3rd ed.,Vol. 5, pp. 51-57). Oxford, UK: Elsevier.

Sammons, P., Anders, Y., Sylva, K., Melhuish, E., Siraj-Blatchford, I., Taggart, B., \& Barreau, S. (2008). Children's cognitive attainment and progress in English primary schools during key stage 2: Investigating the potential continuing influences of pre-school education. Zeitschrift für Erziehungswissenschaft, Sonderheft, 1, 179-198.

Sammons, P., Elliot, K., Sylva, K., Melhuish, T., Siraj-Blatchford, I., \& Taggart, B. (2004). The impact of pre-school on young children's cognitive attainments at entry to reception. British Educational Research Journal, 30(5), 691-712. doi:10.1080/0141192042000234656

Sammons,P.,Sylva,K.,Melhuish,E.,Siraj-Blatchford,I.,Taggart,B.,Draghici,D., \& Smees,R.(2011). Effective Pre-School, Primary and Secondary Education Project (EPPSE 3-14): Influences on students' development in Key Stage 3: Social-behavioural outcomes in Year 9 (Full report). London: Institute of Education, University of London.

Sammons, P., Sylva, K., Melhuish, E., Siraj-Blatchford, I., Taggart, B., \& Hunt, S. (2008). Effective Preschool and Primary Education 3-11 Project (EPPE 3-11): Influences on children's attainment and progress in Key Stage 2: Cognitive outcomes in Year 6 (Research Report No. DCSF-RR048). Nottingham: Department for Children, Schools and Families.

Sammons, P., Sylva, K., Melhuish, E., Siraj-Blatchford, I., Taggart, B., \& Jelicic, H. (2008). Effective Pre-school and Primary Education 3-11 Project (EPPE 3-11): Influences on children's development and progress in Key Stage 2: Social/behavioural outcomes in Year 6 (Research Report No. DCSFRR049). Nottingham: Department for Children, Schools and Families.

Sammons, P., Sylva, K., Melhuish, E., Siraj-Blatchford, I., Taggart, B., Toth, K. \& Smees, R. (2011). Effective Pre-school, Primary and Secondary Education Project (EPPSE 3-14): Influences on students' attainment and progress in Key Stage 3: Academic outcomes in English, maths and science in Year 9 (Full report). London: Institute of Education, University of London.

Sammons, P., Sylva, K., Melhuish, E.C., Siraj, I., Taggart, B., Toth, K., \& Smees R. (2014). Effective Preschool, Primary and Secondary Education 3-16 Project (EPPSE 3-16): Influences on students' GCSE attainment and progress at age 16 (Research Report No. RR352). London: Department for Education.

Sammons, P., Toth,K., \& Sylva,K.,(2015).Pre-school and early home learning effects on A-level outcome (Research Report). London: Department for Education. 
Sammons, P., Toth, K., \& Sylva, K. (2018). The drivers of academic success for "bright" but disadvantaged students: A longitudinal study of AS and A-level outcomes in England. Studies in Educational Evaluation, 57, $31-41$. doi:10.1016/j.stueduc.2017.10.004

Sammons, P., Toth, K., Sylva, K., Melhuish, E., Siraj, I., \& Taggart, B. (2015). The long-term role of the home learning environment in shaping students' academic attainment in secondary school. Journal of Children's Services, 10(3), 189-201. doi:10.1108/JCS-02-2015-0007

Sénéchal, M., \& LeFevre, J.-A. (2002). Parental involvement in the development of children's reading skill: A five-year longitudinal study. Child Development, 73(2), 445-460. doi:10.1111/1467-8624. 00417

Son, S.-H., \& Morrison, F. J. (2010). The nature and impact of changes in home learning environment on development of language and academic skills in preschool children. Developmental Psychology, 46(5), 1103-1118. doi:10.1037/a0020065

Sy, S. R., Gottfried, A. W., \& Gottfried, A. E. (2013). A transactional model of parental involvement and children's achievement from early childhood through adolescence. Parenting: Science and Practice, 13(2), 133-152. doi:10.1080/15295192.2012.709155

Sylva, K., Melhuish, E., Sammons, P., Siraj-Blatchford, I., \& Taggart, B. (Eds.). (2010). Early childhood matters: Evidence from the effective pre-school and primary education project. Abingdon: Routledge.

Sylva, K. (2013). Enabling parents to support their children's learning at home. European Child \& Adolescent Psychiatry, 22 (Suppl 2), S132.

Totsika, V., \& Sylva, K. (2004). The home observation for measurement of the environment revisited. Child and Adolescent Mental Health, 9(1), 25-35. doi:10.1046/j.1475-357X.2003.00073.x

Vandell, D. L., Belsky, J., Burchinal, M., Steinberg, L., \& Vandergrift, N. (2010). Do effects of early child care extend to age 15 years? Results from the NICHD study of early child care and youth development. Child Development, 81(3), 737-756. doi:10.1111/j.1467-8624.2010.01431.x

Van Steensel, R. (2006). Relations between socio-cultural factors, the home literacy environment and children's literacy development in the first years of primary education. Journal of Research in Reading, 29(4), 367-382. doi:10.1111/j.1467-9817.2006.00301.x 